Aus dem Institut für Diagnostische und Interventionelle Neuroradiologie (Prof. Dr. med. C.-H. Riedel)

der Medizinischen Fakultät der Universität Göttingen

\title{
Wert des CBV-ASPECTS im Vergleich zum CTA-ASPECTS bei Patienten mit akutem ischämischem Schlaganfall
}

\author{
INAUGURAL-DISSERTATION \\ zur Erlangung des Doktorgrades \\ der Medizinischen Fakultät der \\ Georg-August-Universität zu Göttingen
}

vorgelegt von

Ioannis Tsogkas

aus

Marousi, Griechenland

Göttingen 2020 
Dekan:

Referent/in:

Ko-Referent/in:

Datum der mündlichen Prüfung:
Prof. Dr. W. Brück

Prof. Dr. M.-N. Psychogios

Prof. Dr. T.-R. Döppner

03.12 .2020 
Hiermit erkläre ich, die Dissertation mit dem Titel "Wert des CBV-ASPECTS im Vergleich zum CTA-ASPECTS bei Patienten mit akutem ischämischem Schlaganfall" eigenständig angefertigt und keine anderen als die von mir angegebenen Quellen und Hilfsmittel verwendet zu haben.

Göttingen, den .............. 
Die Daten, auf denen die vorliegende Arbeit basiert, wurden teilweise publiziert:

Tsogkas I, Knauth M, Schregel K, Behme D, Wasser K, Maier I, Liman J, Psychogios MN (2016): Added value of CT perfusion compared to CT angiography in predicting clinical outcomes of stroke patients treated with mechanical thrombectomy. Eur Radiol 26, 4213-4219

Die Ergebnisse dieser wurden im Rahmen der 50. Jahrestagung der „Deutschen Gesellschaft für Neuroradiologie e.V.“ 2015 in Köln als Postervortrag vorgestellt (Abstracts 50. Jahrestagung der Deutschen Gesellschaft für Neuroradiologie 15.-17. Oktober 2015 Gürzenich, Köln). 


\section{Inhaltsverzeichnis}

Abbildungsverzeichnis ..........................................II

Tabellenverzeichnis ............................................III

Abkürzungsverzeichnis ..........................................IV

1. Einleitung ..........................................................1

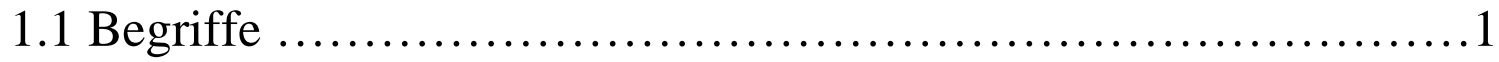

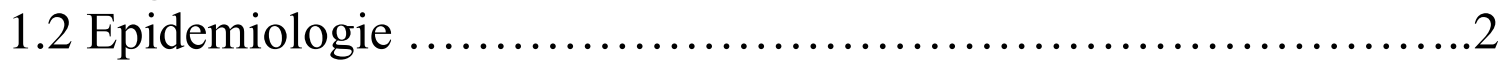

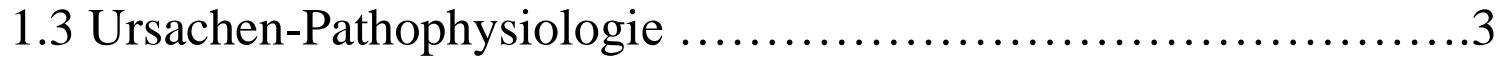

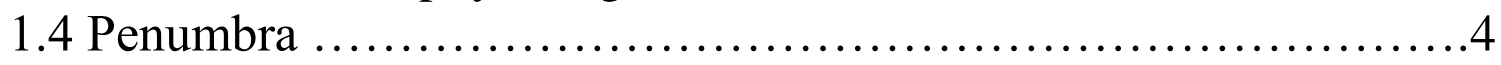

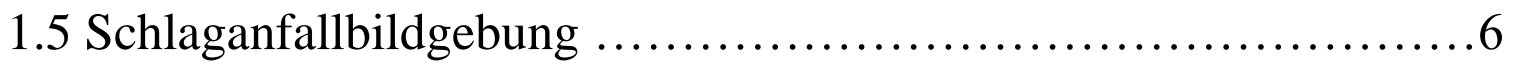

1.5.1 Diagnostik ..........................................6

1.5.2 Vergleich der Schlaganfallbildgebung mittels CT und MRT ................................. 7

1.5.3 Native Computertomographie ......................... 8

1.5.4 Infarktfrühzeichen ................................... 8

1.5.5 ASPECTS …...................................... 10

1.5.6 Multimodale Bildgebung ...............................13

1.6 Therapie .................................................. 14

1.7 Studien und Patientenselektion ................................ 15

2. Patientenkollektiv und Methoden ...............................17

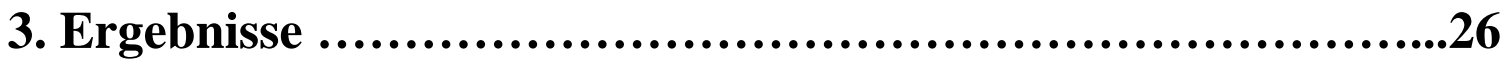

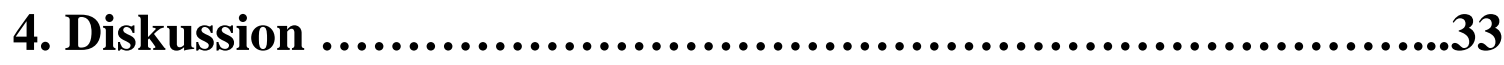

5. Zusammenfassung ..........................................42

6. Literaturverzeichnis .........................................43

7. Kopie der Publikation ...........................................48 


\section{Abbildungsverzeichnis}

Abbildung 1: Infarktkern, Penumbra und benigne Oligämie

Abbildung 2: Aufhebung der Rinden-Mark-Differenzierbarkeit

Abbildung 3: Dichteminderung der Basalganglien

Abbildung 4: ASPECTS-Score 11

Abbildung 5: Hyperdenses Arterienzeichen 12

Abbildung 6: Vergleich der ROC-Kurvenanalysen 31

Abbildung 7: NCCT-, CTA-, und CBV-ASPECTS einer 75-jährigen

Patientin 36 


\section{Tabellenverzeichnis}

Tabelle 1: Todesfälle in Deutschland infolge eines Schlaganfalls ..........3

Tabelle 2: mTICI-Graduierung .................................... 18

Tabelle 3: Modifizierte Rankin-Skala .................................24

Tabelle 4: Baseline-Charakteristika, Zeiten und klinisches Ergebnis ......29

Tabelle 5: Retrospektive Prädiktion des klinischen Ergebnisses............30 


\section{Abkürzungsverzeichnis}

\begin{tabular}{|c|c|}
\hline $\mathrm{ACM}$ & Arteria cerebri media \\
\hline $\mathrm{CBF}$ & cerebral blood flow, zerebraler Blutfluss \\
\hline $\mathrm{CBV}$ & cerebral blood volume, zerebrales Blutvolumen \\
\hline $\mathrm{CT}$ & Computertomographie \\
\hline CTA & CT-Angiographie \\
\hline CTP & CT-Perfusion \\
\hline ICC & intraclass correlation coefficient, Intra-Klassen-Korrelation (IKK) \\
\hline IQR & interquartile range, Interquartilsabstand \\
\hline $\mathrm{mRS}$ & modified Rankin Scale, modifizierte Rankin-Skala \\
\hline MRT & Magnetresonanztomographie \\
\hline MSCT & Mehrschicht-Detektor-Geräte \\
\hline mTICI & $\begin{array}{l}\text { modified thrombolysis in cerebral infarction, modifizierte Thrombolyse im } \\
\text { zerebralen Infarkt }\end{array}$ \\
\hline MTT & mean transit time, mittlere Transitzeit \\
\hline NCCT & noncontrast $C C T$, native Computertomographie des Kopfes \\
\hline NIHSS & National Institutes of Health Stroke Scale, Schlaganfallskala des National \\
\hline
\end{tabular}


rt-PA recombined tissue type plasminogen activator, rekombinanter gewebespezifischer Plasminogenaktivator

SAB subarachnoidale Blutung

SOP $\quad$ standard operating procedure, Standardvorgehensweise

SVT Sinus-Venen-Thrombose

TDC time density curve, Zeit-Dichte-Kurve

TTD time to drain, Zeitwert bis zur niedrigsten Kontrastmittelkonzentration nach KM-Gabe

TTP time to peak, Zeitwert bis zur höchsten Kontrastmittelkonzentration 


\section{Einleitung}

Als ischämischer Schlaganfall wird eine „schlagartig“ auftretenden Durchblutungsstörung des Gehirns bezeichnet, die aufgrund des Sauerstoff- und Glukosemangels zu einer Nekrose des betroffenen Gehirngewebes führen kann. Der Schlaganfall ist eine der häufigsten Todesursachen in Deutschland und weltweit die häufigste Ursache für Langzeitbehinderung. Am häufigsten liegt ein Verschluss eines hirnversorgenden Gefäßes (ischämischer Schlaganfall) zugrunde, der sowohl medikamentös als auch interventionell behandelt werden kann. Trotzdem profitieren nicht alle Patienten von der Therapie. In den letzten Jahren wurden mehrere Prädiktoren des klinischen Ergebnisses der Patienten mit akutem ischämischem Schlaganfall, die interventionell behandelt wurden, vorgeschlagen und entwickelt. In dieser Studie vergleichen wir zwei häufig verwendete Prädiktoren (CTAngiographie - Alberta stroke program early CT score [CTA-ASPECTS] und cerebral blood volume - Alberta stroke program early CT score [CBV-ASPECTS]) und analysieren ihre Bedeutung in der Schlaganfalltherapie.

\section{$\underline{1.1 \text { Begriffe }}$}

Laut der DGN (Deutsche Gesellschaft für Neurologie - 2016) ist der Schlaganfall: „ein akutes fokales neurologisches Defizit aufgrund einer umschriebenen Durchblutungsstörung des Gehirns“. Die Thrombektomie wird bei einem Hauptstammverschluss neben der medikamentösen Therapie zur Behandlung des akuten ischämischen Schlaganfalls empfohlen. Als Thrombektomie bezeichnet man die endovaskuläre Rekanalisation eines Verschlusses der hirnversorgenden Arterien mithilfe 
von Kathetern und unter Röntgen-Darstellung. In Deutschland wird die Thrombektomie in der Regel vom Neuroradiologen durchgeführt.

\section{$\underline{1.2 \text { Epidemiologie }}$}

Im Jahr 2012 war laut Statistischem Bundesamt der Schlaganfall nach Herz- und Krebserkrankungen die dritthäufigste Todesursache und die häufigste Ursache für bleibende Behinderung im Erwachsenalter. Die 12-Monats-Prävelenz eines Schlaganfalls oder chronischer Beschwerden infolge eines Schlaganfalls in den letzten 12 Monaten in Deutschland liegt bei Personen im Alter von 75 Jahren und älter bei 6,4\% bei Frauen und 6,1\% bei Männern (12-Monats-Prävalenz von Schlaganfall oder chronischen Beschwerden infolge eines Schlaganfalls in Deutschland). In Anbetracht einer immer älter werdenden Bevölkerung steigt jedes Jahr die Anzahl der Schlaganfälle, die Anzahl der Todesfälle aufgrund eines Schlaganfalls nimmt jedoch ab (Tab. 1, ). Für 2006 stellt das Statistische Bundesamt 28.566 Todesfälle (18.605 Frauen) infolge vom Schlaganfall und für 2015 18.883 (12.078 Frauen) Todesfälle fest. Die Grundlage dieser positiven Entwicklung sind die Fortschritte sowohl in der Diagnostik als auch in der Therapie. 
Tab. 1: Abnehmende Anzahl der Todesfälle in Deutschland aufgrund eines Schlaganfalls in den Jahren 2006 bis 2015 (eigene Tabelle, Daten: Statistisches Bundesamt, www-genesis.destatis.de).

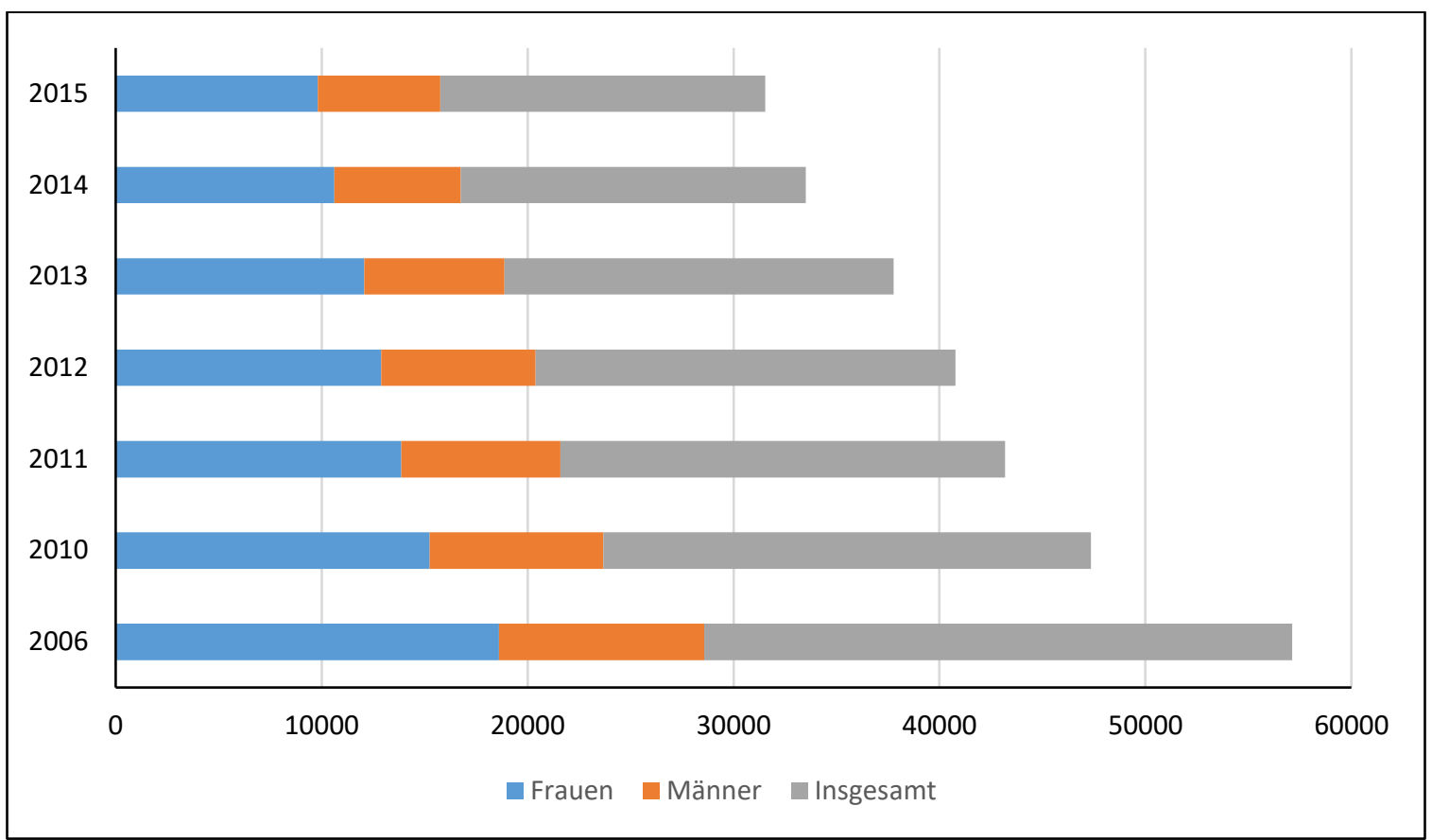

\section{$\underline{1.3 \text { Ursachen-Pathophysiologie }}$}

Der ischämische Schlaganafall, der durch einen Verschluss eines Hirngefäßes verursacht wird, ist der häufigste Typ (80\%, Robert Koch Institut November 2015). Andere Ursachen eines Schlaganfalls sind die intraparenchymale Blutung, die Sinus-Venen-Thrombose (SVT) sowie die subarachnoidale Blutung (SAB).

Bei einer zerebralen Ischämie liegt eine signifikante Minderung des Blutflusses (cerebral blood flow, CBF) einer Region zugrunde. Der zerebrale Blutfluss in der menschlichen, gesunden grauen Substanz liegt bei $60-70 \mathrm{ml} / 100 \mathrm{~g} / \mathrm{min}$. Bei einer Minderung des CBF unter $20 \mathrm{ml} / 100 \mathrm{~g} / \mathrm{min}$ sind die geschädigten Nervenzellen aufgrund der kollateralen Versorgung funktionell intakt und können sich bei erfolgreicher Reperfusion vollständig erholen. Eine weitere Senkung unter $10 \mathrm{ml} / 100 \mathrm{~g} / \mathrm{min}$ führt zur Nekrose der Zellen, die dem 
Infarktkern entspricht. In einem Areal mit CBF-Werten über $20 \mathrm{ml} / 100 \mathrm{mg} / \mathrm{min}$ und weniger als $50 \mathrm{ml} / 100 \mathrm{mg} / \mathrm{min}$ besteht eine Minderversorgung (benigne Oligämie), die aufgrund der Autoregulation der zerebralen Gefäße zu keinen neurologischen Defiziten führt.

\section{$\underline{1.4 \text { Penumbra }}$}

Der Begriff der ,ischämischen Penumbra“ wurden in den 1970er Jahren eingeführt. Dieser Begriff entspricht der umgebenden Zone des Infarktkerns mit CBF-Werten unter 20 $\mathrm{ml} / 100 \mathrm{~g} / \mathrm{min}$, aber über $10 \mathrm{ml} / 100 \mathrm{~g} / \mathrm{min}$. Das sogenannte tissue at risk ist eine Hirnregion mit geschädigten aber noch funktionell intakten Neuronen. Bis zur Publikation der MR CLEAN-Studie im Januar 2015 (Berkhemer et al. 2015) war das Ausmaß des ,tissue at risk“ ein wesentlicher Einflussfaktor für die Entscheidung, ob eine Thrombektomie durchgeführt werden sollte. Nach der Reperfusion des ischämischen Areales (Penumbra und benigne Oligämie) normalisieren sich die CBF-Werte und ein Progress des Infarktkerns wird verhindert (Abb. 1). Seit 2015 und nach der Publikation der letzten Studien wird das Ausmaß der Penumbra zur Selektion der Patienten, die bis zu 6 Stunden nach Auftreten der Symptome endovaskulär behandelt werden können, immer weniger verwendet. Zugrunde liegt die Metaanalyse der Subgruppen der in dieser Studie endovaskulär behandelten Patienten, die beweisen konnte, dass unabhängig vom Ausmaß dieses Areals Patienten von der Thrombektomie profitieren können. In unserer Klinik wird die Penumbra zur Selektion der Patienten innerhalb von 6 Stunden nach Symptombeginn prinzipiell nicht verwendet. Allerdings gibt es weiterhin viele Zentren, die die Bestimmung der Penumbra auch in diesem Zeitfenster als essenziell betrachten, und viele Studien, die dies belegen (Campbell et al. 2015; Saver et al. 2015). In Anbetracht der aktuellen Leitlinie 
sollten erweiterte Bildgebungsparameter, wie die Bestimmung der Penumbra, herangezogen werden, um Patienten mit potenziell zu rettendem Gewebe zu identifizieren, die später als 6 Stunden nach Symptombeginn behandelt werden (Deutsche Gesellschaft Neurologie - Leitlinien für Diagnostik und Therapie in der Neurologie, Akuttherapie des ischämischen Schlaganfalls - Ergänzung 2015).

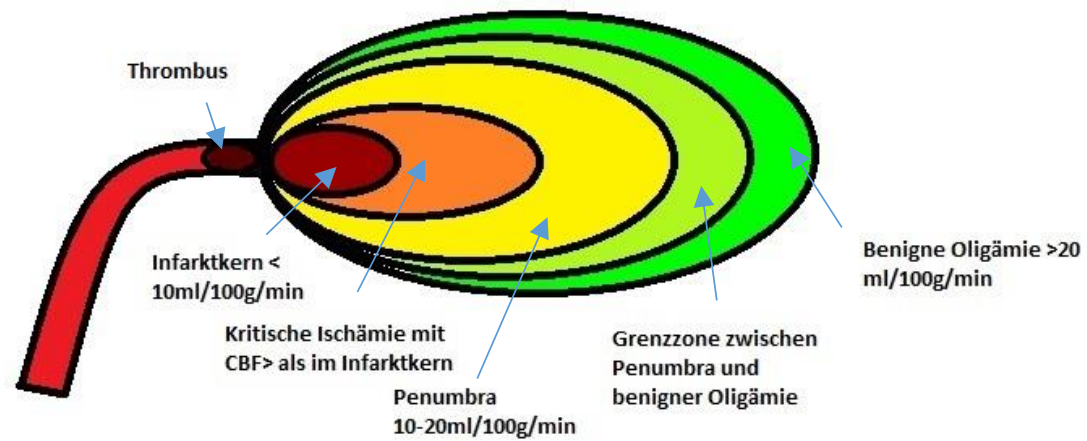

Abb. 1: Das Bild zeigt die Regionen distal des arteriellen Verschlusses mit Grenzzonen zwischen Infarktkern, Penumbra und benigner Oligämie. Diese Regionen verändern sich im Zeitverlauf, und der Infarktkern nimmt zu. Der Verlauf wird von Änderungen der leptomeningealen Kollateralen und toxisch metabolischen und physiologischen Prozessen beeinflusst (eigene Abbildung).

Ein wesentliches Prinzip der Schlaganfalltherapie wird in dem Satz „Time is Brain“ (Zeit ist Hirn) zusammengefasst, da das Hirngewebe schnell und irreversibel geschädigt wird im Falle einer Sauerstoffunterversorgung. Für das Erreichen eines optimalen Behandlungsergebnisses ist daher eine möglichst kurze Zeit bis zur Reperfusion anzustreben. Diese Zeitdauer wird von verschiedenen Faktoren beeinflusst, dabei sind die 
frühe Erkennung des Schlaganfalls, der rasche Transport der Patienten ins Krankenhaus, die schnelle Diagnose und die Rekanalisation des Verschlusses essenziell. Insbesondere spielt die Bildgebung des Schlaganfalls eine entscheidende Rolle im Ausschluss einer intrakraniellen Blutung als Ursache der Symptome, im Nachweis des Verschlusses des hirnversorgenden Gefäßes und im Falle eines unklaren Symptombeginns in der Selektion der Patienten, die von einer Thrombektomie profitieren könnten.

\section{$\underline{1.5 \text { Schlaganfallbildgebung }}$}

\subsubsection{Diagnostik}

Die Schlaganafalldiagnostik erfolgt sowohl mittels Computertomographie (CT), als auch mittels Magnetresonanztomographie (MRT). Die schnelle Durchführung der Diagnostik ist von fundamentaler und entscheidender Bedeutung. Durch die bildgebende Diagnostik müssen vier entscheidende Fragen beantworten werden, damit die weiteren Behandlungsschritte entschieden werden können: Besteht eine intrakranielle Blutung? Liegt ein akuter Gefäßverschluss vor, der lysiert oder endovaskulär behandelt werden könnte? Falls vorhanden, wie ausgedehnt ist der Infarktkern? Ist das vom Verschluss betroffene Areal größer als der Infarktkern? Mittels spezieller Untersuchungstechniken, die im Verlauf der Jahre immer besser und schneller geworden sind, kann man die vier oben genannten Fragen antworten.

\subsubsection{Vergleich der Schlaganfallbildgebung mittels CT und MRT}

Ein großer Vorteil der CT ist die breitere Verfügbarkeit. Darüber hinaus bestehen ein geringerer Aufwand bei der Patientenlagerung und ein besseres Monitoring während der 
Untersuchung. Mittels CT können sowohl instabile Patienten als auch Patienten mit MRTKontraindikationen (z.B. Schrittmacheraggregat) problemlos untersucht werden. Ein klarer Nachteil der CT ist die Verwendung ionisierender Strahlung sowie die Notwendigkeit der Gabe vom jodhaltigen Kontrastmittel, welche bei wiederholten Untersuchungen oder bei Patienten unter Metformintherapie in Betracht gezogen werden muss. Wie oben beschrieben muss vor dem Beginn einer Lyse-Therapie eine Blutung ausgeschlossen werden. Kidwell et al. konnten in den letzten Jahren zeigen, dass die MRT mithilfe von blutungssensitiven Sequenzen für den Nachweis akuter Blutungen mindestens so sensitiv ist, wie die CT (Kidwell et al. 2004). Ein entscheidender Vorteil der MRT ist die sichere Abbildung eines Infarktes unabhängig von der Größe oder der Zeit nach Symptombeginn (dem Alter der Ischämie-Läsion). Die Schlaganfallbildgebung mittels multimodaler CT dauert einschließlich der Auswertung ca. 10-15 Minuten (Handschu et al. 2004). Ein komplettes Schlaganfallprotokoll in Kernspintomographie dauert mindestens 20 Minuten. In Anbetracht der 2016 aktualisierten Leitlinien der Akuttherapie des ischämischen Schlaganfalls gibt es keine klare Empfehlung in Bezug auf die Methode der Wahl. Die Wahl der Methode bleibt dem jeweiligen Zentrum und dessen standard operating procedure (SOP) überlassen, sollte jedoch zu keiner zeitlichen Verzögerung führen. 


\subsubsection{Native Computertomographie}

Mittels nativer Computertomographie (CT), die die weltweit am weitesten verbreitete Methode zur bildgebenden Diagnostik beim Schlaganfall ist, sollen zuerst eine intrakranielle Blutung sowie ein großer demarkierter Infarkt ausgeschlossen werden, damit die systemische intravenöse Thrombolyse eingesetzt werden kann (vorausgesetzt, dass der Gefäßverschluss nicht länger als 4,5 Stunden besteht und keine weiteren Kontraindikationen gegen die Anwendung der systemischen Lyse vorliegen).

\subsubsection{Infarktfrühzeichen}

Die frühen Ischämiezeichen (early ischemic signs) sind bildmorphologische Zeichen eines akuten Infarktes. Durch das frühe ischämische zytotoxische Ödem (Wassereinstrom) lässt sich innerhalb der ersten 4 Stunden eine lokale Dichteminderung des Gewebes im nativen CT erkennen, die zu einer Frühhypodensität des Rindenbandes und der Basalganglien führt. Diese Hypodensität korreliert gut mit dem irreversibel geschädigten Infarktareal (Abb. 2 und 3) (Gonzalez 2006; Kucinski 2005). Die ausgedehnten Frühinfarktzeichen sind ein guter Prädiktor für ein schlechtes klinisches Outcome (von Kummer et al. 1994) und unter Umständen auch für das erhöhte Risiko einer symptomatischen intrazerebralen Blutung (Larrue et al. 2001). 


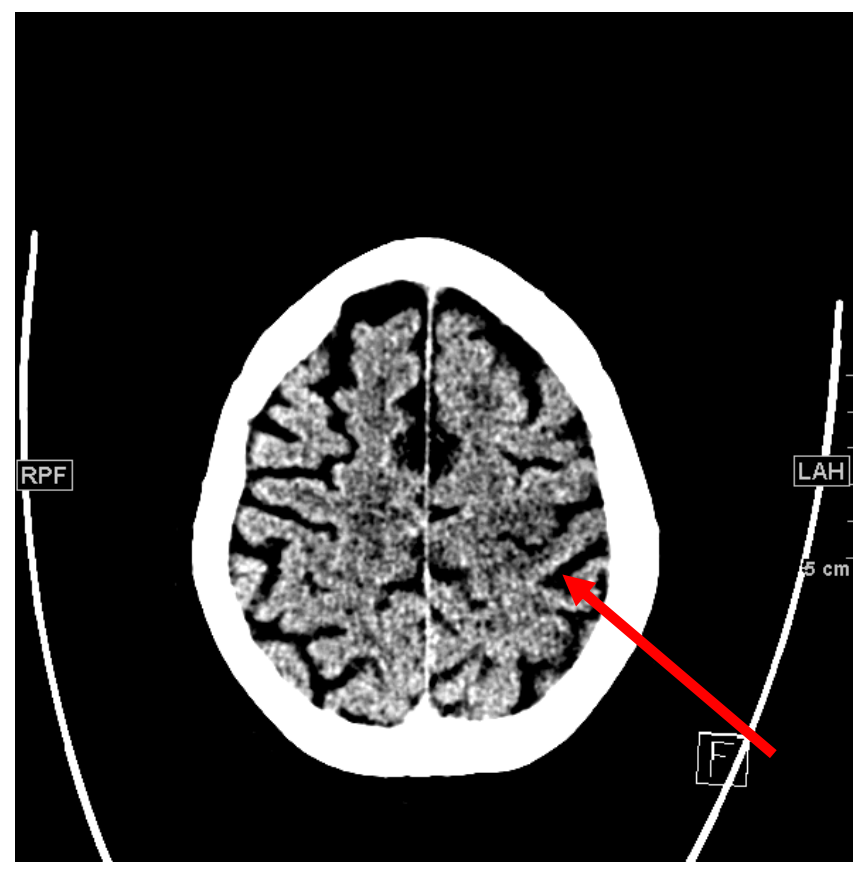

Abb. 2: In diesem Bild zeigt sich eine Aufhebung der Rinden-Mark-Differenzierbarkeit um den linken Sulcus centralis (Pfeil).

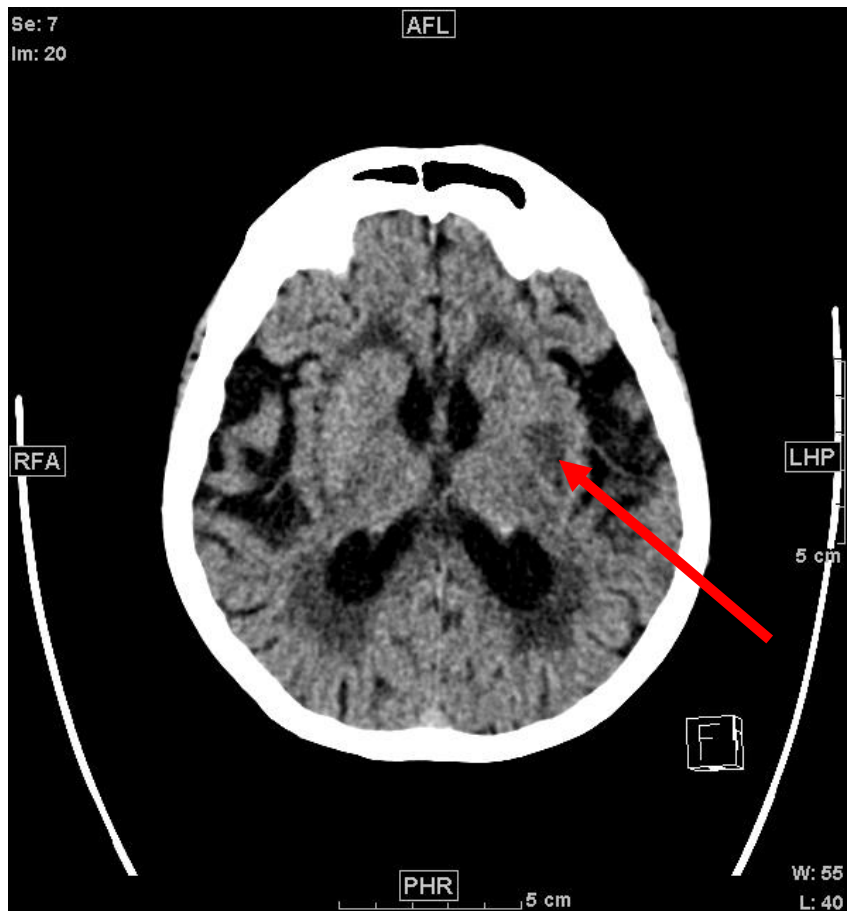

Abb. 3: In den linken Basalganglien lässt sich eine Dichteminderung des linken Putamens erkennen (Pfeil). 


\subsubsection{ASPECTS}

Studien haben die Erkennbarkeit der Infarktfrühzeichen im nativen CT untersucht und gezeigt, dass die Sensitivität und die Übereinstimmung verschiedener Untersucher zwischen 20 und 87\% bzw. 45 und 78\% liegen (Wardlaw und Mielke 2005). Der Alberta Stroke Program Early CT Score (ASPECTS) ist ein 10-Punkte (semi-)quantitativer topographischer CT Scan Score (Auswertung) der Ausdehnung der Infarktfrühzeichen. Er wurde entwickelt, um eine einfach reproduzierbare Evaluation der Ausdehnung der frischen ischämischen Läsionen in der nativen präinterventionellen CT zu bieten. Er ist verwendbar bei Patienten mit ischämischem akuten Schlaganfall des Stromgebietes der Arteria cerebri media. Ergebnisse von mehreren Studien haben gezeigt, dass das mit ASPECTS ausgewertete Ausmaß der ischämischen Frühzeichen im präinterventionellen nativen CT, vor der jeweiligen Therapie, das funktionelle Ergebnis oder das Risiko für posttherapeutische intrakranielle Blutung prognostizieren kann (Barber et al. 2000; Mak et al. 2003). In Anbetracht aber der letzten Metaanalyse der HERMES Gruppe (Goyal et al. 2016) lässt sich der Ausmaß der Infarktfrühzeichen mit der hämorrhagischen Transformation nicht korrelieren. 


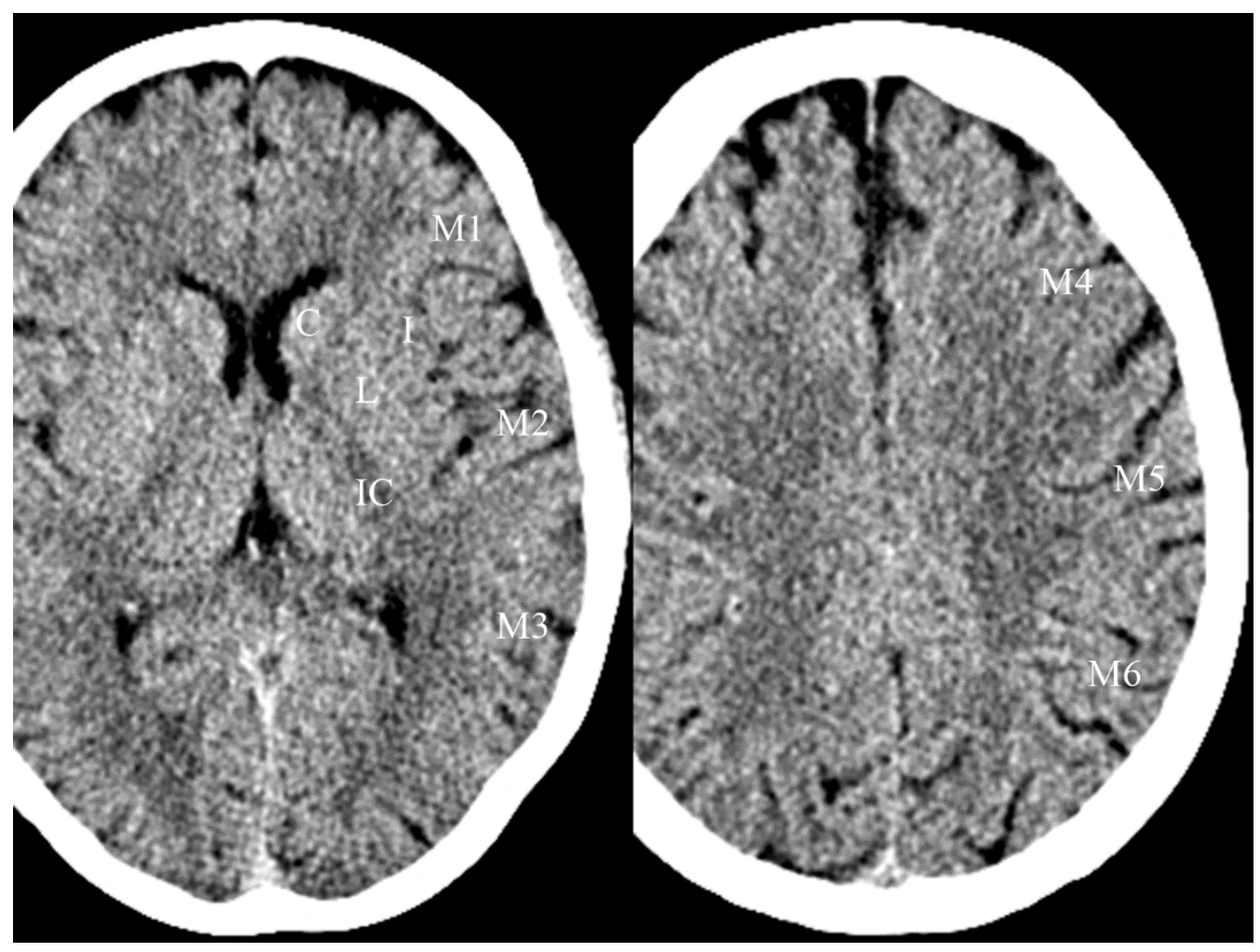

Abb. 4: Das linke Versorgungsgebiet des Arterie cerebri media ist in 10 Regionen eingeteilt. Je größer die Demarkation (d. h. Hypodensität) eines Infarktes, desto kleiner der ASPECTS-Score. Daten aus Tsogkas et al. (2016), mit freundlicher Genehmigung von Springer Nature, licence number:4923090199089 (entspricht Abbildung 1).

Die Auswertung des ASPECTS betrifft zwei Regionen des Mediastromgebietes (Versorgungsgebietes des A. cerebri media), das Niveau der Basalganglien, wo Thalamus, Basalganglien und Nucleus caudatus erkennbar sind und das supraganglionische Niveau, wo sich Corona radiata und centrum semiovale abbilden. Das Mediastromgebiet lässt sich in zehn Punkten unterteilen. Die capsula interna, der Nucleus caudatus und der Linsenkern sind drei Punkte und der Kortex, einschließlich des insulären Kortex, die übrigen sieben Punkte (Abb. 4). Für jedes demarkierte Areal wird ein Punkt abgezogen. Eine Punktwert 
von zehn Punkten entspricht einem Befund ohne Zeichen eines Infarktes. Im Gegenteil ein Punktwert von null Punkten entspricht einem komplett demarkierten Mediainfarkt.

Die einfache Reproduzierbarkeit führt $\mathrm{zu}$ einer niedrigen inter- und intraobserver Variabilität (Pexman et al. 2001) (Barber et al. 2000) (Coutts et al. 2004). Wie oben beschrieben, wurde in mehreren Studien gezeigt, dass der ASPECTS das posttherapeutische klinische Ergebnis prognostizieren kann und deswegen für die Selektion der Patienten, bei denen eine endovaskuläre Therapie durchgeführt werden sollte, angewendet wurde. Dieser Prognosewert wurde sowohl für die intravenöse Therapie als auch für die endovaskuläre Therapie gezeigt (Hill et al. 2014).

Ein weiteres Infarktfrühzeichen ist das hyperdense Arterienzeichen, das ein indirekter Hinweis auf den Thrombus sein kann (Dichteanhebung des Gefäßes, hyperdense middle cerebral artery sign). Das Gefäß bildet sich aufgrund der Thrombosierung hyperdenser ab (Abb. 5).

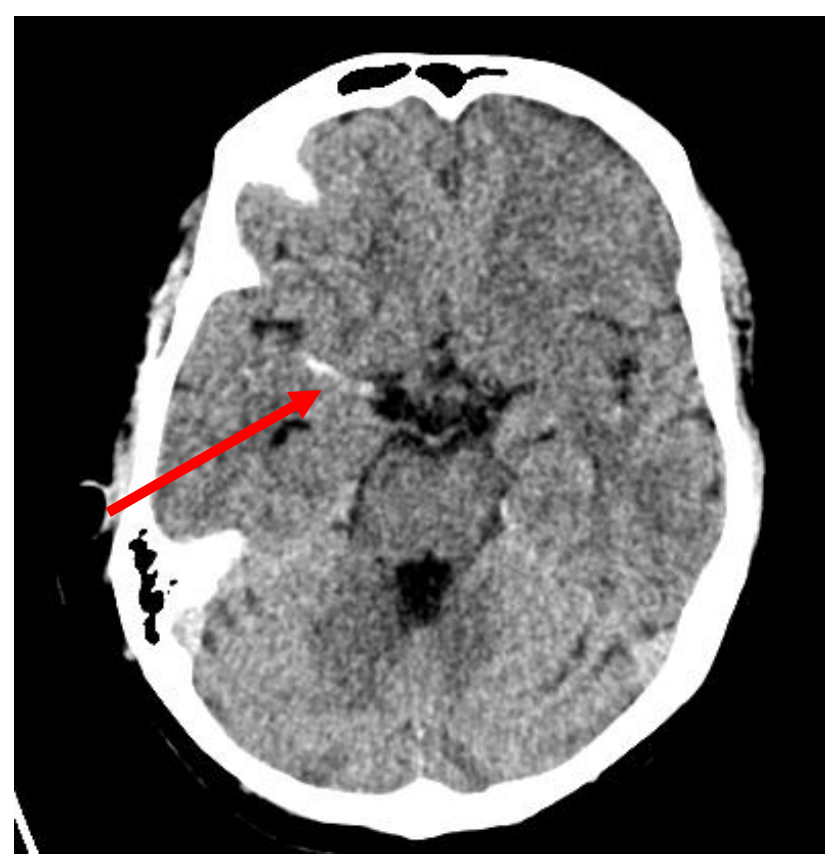


Abb. 5: In diesem nativen CT (ohne Kontrastmittelgabe) des Kopfes und im Vergleich zur Gegenseite bildet sich die rechte Arterie cerebri media hyperdens ab. Diese Hyperdensität entspricht dem Thrombus, der den Gefäßverschluss verursacht (Pfeil).

\subsubsection{Multimodale Bildgebung}

Mit der Hilfe der Mehrschicht-Detektor-Geräte (MSCT) ist die Beurteilung sowohl der intrakraniellen, als auch der extrakraniellen Gefäße möglich. Der Ausschluss oder die Bestätigung eines Gefäßverschlusses und dessen Lokalisation sind Fragen, die durch eine CTA beantwortet werden können. Im Fall eines akuten Ereignisses ist die Beurteilung des Gefäßstatus von großer Bedeutung, da im Falle eines nachgewiesenen Verschlusses die endovaskuläre Behandlung erwogen werden muss. Darüber hinaus wurde beschrieben, dass die Veränderungen in den Quellbildern der CTA (source images, CTA-SI), die mit älteren CT-Geräten durchgeführt wurden, dem Infarktkern entsprechen. Diese Veränderungen korrelieren gut mit den Veränderungen der Diffusion-Sequenz und der MR-Perfusionsbildgebung, die die sensitivsten Methoden zur Bestätigung des Infarktes und dessen Kerns sind. (Schramm et al. 2004).

Die CT-Perfusion ist eine dynamische Computertomographie und sie gibt, wie die MRPerfusion, Informationen über die Blutversorgung des Gehirns. Die Durchführung hängt von zwei mathematischen Modellen ab, die Dekonvolutionstechnik und das Gradientenmodell (Wiesmann et al. 2004). Aus der Zeit-Dichte-Kurve (time density curve, TDC) der CT-Perfusion werden für jedes Voxel die folgenden hämodynamischen Parameter errechnet (Trenkler 2008):

Das zerebrale Blutvolumen (CBV, cerebral blood volume) ist bezeichnet als das Gesamtvolumen des Blutes pro Volumeneinheiten vom Hirngewebe. Die Einheit ist 
ml/100g und beträgt bei gesundem Gewebe für die graue Substanz etwa $4 \mathrm{ml} / 100 \mathrm{~g}$, für die weiße Substanz (Marklager) 2 ml/100g. Der zerebrale Blutfluss (CBF, cerebral blood flow) ist definiert als das Blutvolumen welches pro Zeiteinheit durch ein bestimmtes Hirngewebevolumen fließt. Der CBF wird in der Einheit ml/100g/min angegeben und beträgt bei gesundem Gewebe für die graue Substanz etwa $60 \mathrm{ml} / 100 \mathrm{~g} / \mathrm{min}$ und für die weiße Substanz $25 \mathrm{ml} / 100 \mathrm{~g} / \mathrm{min}$. Die mittlere Transitzeit (MTT, mean transit time) ist die durchschnittliche Zeit, die das Blut in einem bestimmten Hirnareal vom arteriellen Inflow bis zum venösen Outflow benötigt. Die MTT wird durch die Formel MTT $=\mathrm{CBV} / \mathrm{CBF}$ berechnet. Für die graue Substanz beträgt die MTT 4 Sekunde (s) und für die weiße Substanz 4,8 s. Die time to peak (TTP) ist die Zeit vom Start der Kontrastmittelinjektion bis zum maximalen Kontrastmittelenhancement in einer bestimmten Region. Die time to drain (TTD) ist die Zeit vom maximalen Kontrastenhancement bis zur niedrigsten Kontrastkonzentration.

\subsection{Therapie}

Ein ischämischer Schlaganfall kann medikamentös (intravenöse Thrombolyse) sowie endovaskulär (intraarterielle Thrombolyse) behandelt werden.

Die Sicherheit und die Effektivität des intravenös verabreichten Gewebeplasminogenaktivators (i.v.-rt-PA) innerhalb der ersten 3 Stunden nach ischämischem Schlaganfall wurden in der NINDS-Studie gezeigt, wenn eine Blutung computertomographisch ausgeschlossen wurde ("Tissue plasminogen activator for acute ischemic stroke. The National Institute of Neurological Disorders and Stroke rt-PA Stroke Study Group" 1995). Nach der Publikation der ECASS III - Studie hat sich das Fenster 
der Anwendung der systemischen Lyse von 3 auf 4,5 Stunden erweitert (Hacke et al. 2008).

Innerhalb der ersten 4,5 Stunden und unter gewissen Umständen war die systemische intravenöse Thrombolyse mit rt-PA bis 2015 die Standardtherapie für die Behandlung des akuten ischämischen Schlaganfalls. Die Neuro-Thrombektomie ist die endovaskuläre Entfernung eines Blutgerinnsels (Thrombus) aus einem hirnversorgenden Blutgefäß. Das Verfahren wird mithilfe von Kathetern, Drähten und verschiedenen Devices (z.B. StentRetriever) überwiegend von Neuroradiologen durchgeführt. Technisch ist es bereits seit mehr als zwanzig Jahren möglich, die für die meisten Schlaganfälle verantwortlichen Blutgerinnsel mittels verschiedener Methoden zu bergen. Eine Thrombektomie kann bei geeigneten Schlaganfallpatienten die Chancen auf ein Leben ohne Behinderungen erhöhen.

\section{$\underline{1.7 \text { Studien und Patientenselektion }}$}

Vor der Publikation der MR CLEAN-Studie im Jahr 2015 (Berkhemer et al. 2015) war die Thrombektomie nicht als standard of care etabliert. Zugrunde lag die Publikation von mehreren Studien, die nicht beweisen konnten, dass die Thrombektomie bei Patienten mit akutem ischämischem Schlaganfall zu einem signifikant besseren funktionellen Langzeitergebnis führt. Der Tiefpunkt war die Publikation der als unhappy triad bekannt gewordenen drei Studien im Jahre 2013, die alle drei kein Benefit der interventionellen Behandlung des Schlaganfalls zeigen konnten. (IMS III (Broderick et al. 2013), MR RESCUE (Kidwell et al. 2013) und SYNTHESIS Expansion (Ciccone et al. 2013)). Die Wende kam 2015 mit fünf randomisierten Studien (MR CLEAN (Berkhemer et al. 2015), EXTEND-IA (Campbell et al. 2015), ESCAPE (Goyal et al. 2015), SWIFT PRIME (Saver 
et al. 2015) und REVASCAT (Jovin et al. 2015)), in denen eine frühe Therapie mit einem „Stent-Retriever“ der neusten Generation die Prognose vieler Patienten verbesserte.

Die erste positive Studie (MR CLEAN) zeigte, dass die Katheterbehandlung bei 86,2 Prozent der Patienten das verschlossene Gefäß partiel oder vollständig rekanalisieren konnte. 90 Tage nach dem Ereignis wiesen 67 Prozent der Thrombektomie-Patienten eine klinische Verbesserung auf, aber nur 32,6 Prozent waren nach drei Monaten funktionell unabhängig (modifizierte Rankin-Skala, $\mathrm{mRS} \leq 2$ ).

Um futile Rekanalisationen zu vermeiden, wurde die CT-Perfusion in den letzten Jahren verwendet, um Patienten zu selektieren, die von der Thrombektomie profitieren würden. Bei der MR CLEAN Studie erfolgte die Patientenselektion nur durch die native CT und die CTA. Bis zu diesem Zeitpunkt haben andere Institute (Campbell et al. 2015) sowie wir eine strengere Selektion unter Analyse der CTP durchgeführt. Der Grund liegt an der höheren Sensitivität dieser Methode (CTP) bei dem Nachweis des Infarktkerns (Psychogios et al. 2013). Eine in 2014 publizierte Studie hat gezeigt, dass der CTA-SIASPECTS, verglichen mit dem CBV-ASPECTS, ein gleichwertiger Prädiktor des klinischen Outcomes ist (Lum et al. 2014). Die CTA Bildern, die in dieser Studie analysiert und ausgewertet wurden, sind vom Datensatz der CTP extrahiert worden. Eine Korrelation der Ergebnisse mit dem CTA-ASPECTS von der breiter verfügbaren single-phase-CTA war unklar. In unserer Studie analysieren und vergleichen wir den CTA-ASPECTS und den CBV-ASPECTS als Prädiktoren des klinischen Ergebnisses nach Thrombektomie bei Patienten mit akutem ischämischen Schlaganfall. 


\section{Patientenkollektiv und Methoden}

Alle Patienten mit akutem ischämischen Schlaganfall, die in der Universitätsmedizin Göttingen seit Januar 2011 endovaskulär behandelt worden sind, wurden zuerst retrospektiv und im Verlauf prospektiv in eine Excel basierte Schlaganfall-Datenbank aufgenommen. Diese Datenbank wurde von der Ethikkommission der Universitätsmedizin Göttingen genehmigt (Nr. des Ethikantrages 13/7/15An). Aus dieser Datenbank wurden alle Patienten extrahiert, die die folgenden Kriterien erfüllt haben: Durchführung einer kompletten multimodalen CT Untersuchung zwischen Januar 2011 und Dezember 2014 einschließlich einer nativen CT des Kopfes (NCCT), einer CTP des Hirnparenchyms und einer einphasigen CTA des Kopfes und des Halses; Verschluss des M1 Segmentes der Arteria cerebri media (ACM). Der Befund im initialen Angiogramm sollte einem mTICI (modified thrombolysis in cerebral infarction, Tabelle 2) 0 oder 1 entsprechen; 
Tab. 2: mTICI-Graduierung der Reperfusion nach Thrombektomie.

\begin{tabular}{|c|c|}
\hline mTICI Grade & Definition \\
\hline Grad 0 & Keine Perfusion \\
\hline Grad 1 & $\begin{array}{l}\text { Antegrade Perfusion distal des initialen } \\
\text { Verschlusses, aber limitierte } \\
\text { Kontrastierung der distalen Äste mit } \\
\text { geringer oder langsamer distaler } \\
\text { Reperfusion }\end{array}$ \\
\hline $\operatorname{Grad} 2 \mathrm{a}$ & $\begin{array}{l}\text { Antegrade Reperfusion von weniger als } \\
\text { der Hälfte des Stromgebietes, das von der } \\
\text { Arterie versorgt wird, die initial } \\
\text { verschlossen war. }\end{array}$ \\
\hline $\operatorname{Grad} 2 b$ & $\begin{array}{c}\text { Antegrade Reperfusion von mehr als der } \\
\text { Hälfte des Stromgebietes. }\end{array}$ \\
\hline Grad 3 & $\begin{array}{l}\text { Vollständige antegrade Reperfusion ohne } \\
\text { nachweisbaren Verschluss eines distalen } \\
\text { Astes }\end{array}$ \\
\hline
\end{tabular}

sowie erfolgreiche Rekanalisation des M1 Segmentes der Arteria cerebri media, definiert als mTICI $2 \mathrm{~b}$ oder 3 in den Abschlussserien nach mechanischer Thrombektomie. In den Abschlussserien sollte mindestens die Hälfte des Mediastromgebietes reperfundiert sein. 
Patienten mit zusätzlich proximalem Verschluss der Arteria carotis interna wurden ausgeschlossen. Weitere Ausschlusskriterien waren die inkomplette Bildgebung, Bilddaten mit deutlichen Bewegungsartefakten, Alter $<21$ Jahren alt und spontane Reperfusion des verschlossenen Gefäßes (z.B. als Folge der intravenösen Lyse) im initialen Angiogramm vor der Thrombektomie.

Bei Vorstellung oder Verlegung eines Patienten mit Verdacht auf einen akuten ischämischen Schlaganfall erfolgte zuerst die klinische Untersuchung durch die Kollegen der Abteilung für Neurologie in der Notaufnahme und anschließend die Untersuchung mittels multimodaler Computertomographie.

Die Bildgebung wurde bei allen Patienten im CT-Raum der Abteilung für Neuroradiologie mit einem 128-Zeilen-Multidetektor-Computertomographie-Scanner (Siemens Definition AS+; Siemens Healthcare Sector, Forchheim, Deutschland) durchgeführt und schloss ein natives Computertomogramm des Kopfes, eine ganz Kopf-CT-Perfusion und eine Computertomographie-Angiographie der extrakraniellen und der intrakraniellen Hirnarterien ein.

Die CT-Perfusion bestand aus 30 konsekutiven Spiral-CTs vom Gehirn (96mm in z-Achse, 2 Sekunde Delay nach der Kontrastmittelgabe, 45 Sekunde totale Aufnahmezeit, 80kV, 200mAs und effektive Dosis von ca. 5mSv). Mit einem Fluss von 6 mL/s wurden $36 \mathrm{~mL}$ iodhaltigem Kontrastmittel venös gespritzt und nachfolgend ca. 30mL Kochsalz. Die CTPBilddaten wurden automatisch mit einer Bilddicke von $5 \mathrm{~mm}$ und einem Bildabstand von $3 \mathrm{~mm}$ rekonstruiert (H20f Kernel, 512 Matrix) und danach vom Neuroradiologen mit der Hilfe eines kommerziell verfügbaren Software-Pakets (Volume Perfusion CT Neuro; 
Siemens) weiterbearbeitet. Die Bilddaten wurden automatisch mit Bewegungskorrekturund Rauschminderung-Technik für dynamische Daten berechnet.

Für die CTA $(120 \mathrm{kV}, 120 \mathrm{mAs}, 0,3 \mathrm{~s}$ Rotationszeit, 0.6 pitch, 2 x 64 × $0.6 \mathrm{~mm}$ Kollimation/Einblendung, ca. 3mSv) wurden $45 \mathrm{~mL}$ Kontrastmittel und anschließend 30 mL Kochsalz mit einem biphasischen Protokoll gespritzt. Die CTA wurde mit einem einphasigen Protokoll durchgeführt, und zwar mit Verwendung einer Bolustriggerung im Aortenbogen (100 HU Kontrastschwellenwert; 5 s Delay für bolus watch und 3 s Delay nach Erzielen des Kontrastschwellenwerts). Die Rohdaten wurden multiplanar mit $5 \mathrm{~mm}$ Bilddicke und $3 \mathrm{~mm}$ Bildabstand rekonstruiert.

Gemäß den aktuellen Leitlinien wurden die Patienten, die innerhalb von 4,5 Stunden nach Symptombeginn verlegt wurden und in der nativen CCT keine Blutung oder ausgeprägte Demarkation eines Infarktes aufwiesen, intravenös mit rekombinantem gewebespezifischem Plasminogenaktivator (recombinant tissue plasminogen activator, IV rt-PA) behandelt. Die empfohlene Gesamtdosis beträgt $0,9 \mathrm{mg} / \mathrm{kg}$ Körpergewicht. Sofort nach der Durchführung der nativen CCT wurde intravenös 10\% der Gesamtdosis als Bolus und nach der Komplettierung der Bildgebung als intravenöse Infusion die restliche rt-PA über $60 \mathrm{~min}$ verabreicht.

Nach der Bildgebung wurden die Patienten in den Angiographieraum (Artis Zee, Siemens Healthcare) verlegt und die mechanische Thrombektomie wurde entweder durch Aspiration oder unter Verwendung eines Stent-retriever-Device oder einer Kombination von beiden Devices durchgeführt. Zur Aspiration wurden die Aspirationskatheter Penumbra MAX (Penumbra, Alameda, California) verwendet. Die Stent-retriever-Devices 
waren die folgenden: Aperio (Acandis, Pforzheim, Deutschland), Trevo (Stryker, Mountain View, California) und 3D Separator (Penumbra).

Alle Patienten wurden von einem Team der Abteilung für Anästhesiologie begleitet. Die Intervention fand entweder unter leichter Sedierung (Analgosedierung) oder in Intubationsnarkose statt. Die Art der Narkose wurde entschieden in Abhängigkeit der klinischen Präsentation des Patienten, insbesondere ob die Vitalparamater stabil waren und ob der Patient eine hohe psychomotorische Unruhe zeigte. Nach Lokalanästhesie der Leiste erfolgte die Einlage einer kurzen 8-French-Schleuse in die rechte oder linke Arterie femoralis communis in Seldinger-Technik. Mit der Hilfe eines 0.035" Standarddrahts (Radifocus, Terumo, Tokio, Japan) und eines überlangen diagnostischen Katheters wurde ein 8-French-Führungskatheter in der ipsilateralen proximalen Arterie carotis interna platziert. Ein 0.014" Mikrodraht wurde verwendet für die Sondierung des thrombosierten Gefäßes mit einem 0.021" oder 0.017" Mikrokatheter oder direkt mit dem Aspirationskatheter. Bei der Verwendung eines Stent-retriever-Devices wurde zuerst die Thrombose mit dem Mikrokatheter passiert und das Stent-retriever-Device wurde durch den Mikrokatheter vorgeschoben um distal, im und proximal des Thrombus freigesetzt zu werden. Nach Aufnahme des Thrombus in die Maschen des Stent-retriever-Devices wurde dieser entweder in den Aspirationskatheter oder in den Führungskatheter zurückgezogen. Abschließend wurden diese als Einheit entfernt.

Sofern die mechanische Thrombektomie unter laufender intravenöser Lyse des Patienten erfolgt ist, wurde die restliche rt-PA lokal intraarteriell über den Aspirationskatheter vor oder nach der Rekanalisation (nach Ermessen des behandelnden Arztes) gegeben. Sofern es bei laufender intravenöser Lyse bereits vor Ablauf der Infusion zu einer erfolgreichen 
und vollständigen Reperfusion (mTICI 3) mittels mechanischer Thrombektomie gekommen ist, wurde die Infusion vorzeitig beendet und die restliche rt-PA verworfen. Nach der Einführung der neuen Standardarbeitsanweisung zwischen den Abteilungen Neuroradiologie, Neurologie und Anästhesiologie im März 2014 sollte die Aufnahme eines Patienten im Lysezeitfenster vom Neurologen angekündigt werden und danach sollte der Neuroradiologe die Entscheidung für die Thrombektomie sofort den Anästhesiologen mitteilen. Auf diese Weise stand immer rechtzeitig das Anästhesieteam zur Verfügung.

Die Effekte der oben beschrieben Standardarbeitsanweisung konnten wir in einer retrospektiven Analyse (Schregel et al. 2016) zeigen und publizieren. Es zeigte sich eine signifikante Reduktion der Zeit von Symptombeginn bis zur Rekanalisationen. Diese hat sich durch die Standardarbeitsanweisung von im Median 264 Minuten auf 211 Minuten reduziert. Dieser Effekt lässt sich primär durch eine signifikante Reduktion der Zeit von Aufnahme (hospital admission) zur Leistenpunktion (groin puncture) erklären. Diese wurde von im Median 121 Minuten (Interquartilsabstand_Interquartile Range-IQR, 96 $161 \mathrm{~min})$ auf 64 Minuten (IQR, $54-77 \mathrm{~min})$ fast halbiert $(\mathrm{p}<0,001)$. Der klinische Outcome der endovaskulär behandelten akuten Schlaganfallpatienten war in dem Zeitraum nach der Einführung der Standardarbeitsanweisung ebenfalls signifikant besser. Hier müssen aber auch Einflussfaktoren, wie veränderte Techniken bei der mechanischen Thrombektomie als mögliche Einflussfaktoren diskutiert werden.

Ein(e) Schlaganfall-zertifizierte(r) Neurologe(in) erhob alle neurologischen Scores (wie z. B. National Institutes of Health Stroke Scale (NIHSS), Modified Rankin Scale (mRS), usw.) zu drei Zeitpunkten: Aufnahme im Krankenhaus, Entlassung aus dem Krankenhaus und 90 Tage nach dem Index-Event. Die neuroradiologischen Scores der Bildgebung und 
der Angiographieserien wurden von drei Neuroradiologen im Konsens bewertet. Zwei der Neuroradiologen verfügten über eine Erfahrung von über fünf Jahren in der Neuroradiologie. Die Neuroradiologen bewerteten getrennt die komplette Bildgebung (NCCT, CTP und CTA) mit dem ASPECT-Score.

Da der Aufnahme-NIHSS, das Alter sowie der CBV-ASPECTS in verschiedenen Publikationen als unabhängige statistisch signifikante Prädiktoren für das postinterventionelle klinische Ergebnis identifiziert wurden, entschlossen wir uns zu der Entwicklung eines neuen Score; den Göttinger Schlaganfall Score (GSS-Goettingen Stroke Scale) mit der folgenden Formel: CBV-ASPECTS-(NIHSS/4 + Alter/20). Wir versprechen uns durch die Kombination der Faktoren die Aussagekraft der Vorhersage zu erhöhen.

Die Bewertung der Bildgebung erfolgte durch die Neuroradiologen in einem Abstand von 90 Tagen und in zwei Gruppen. Die erste Gruppe bestand aus den nativen CCT- und den CTA-Bilddaten. Die zweite bestand aus den CTP-Parametern einschließlich der CBV-, CBF-, MTT- und TTD-Karten. Darüber hinaus wurden die Rater gebeten, retrospektiv die futilen Rekanalisationen zu prognostizieren, entweder mit der Hilfe der NCCT- und CTABildgebung oder unter Berücksichtigung der CTP-Parameter. Dies erfolgte ohne Zugriff auf die Patientendaten. CTA-ASPECTS oder CBV-ASPECTS niedriger als 7 war das Hauptkriterium für futile Rekanalisationen. In Fällen von Uneinigkeit geschah eine Vereinbarung durch Übereinstimmung.

Die modifizierte Rankin-Skala (mRS) ist eine standardisierte Maßzahl, die das klinische Ergebnis der Therapie (keine Symptome) bis 6 (Tod) bewertet (Tab. 3). Die Patienten wurden in zwei Gruppen eingeteilt, Patienten mit gutem klinischen Outcome (Follow-up- 
mRS $\leq 2$ ) und Patienten mit schlechtem klinischen Outcome (Follow-up-mRS in 90 Tagen $>2)$.

Tab. 3: Modifizierte Rankin-Skala

\begin{tabular}{|l|l|}
\hline mRS & Zustand \\
\hline 0 & Keine Symptome \\
\hline 1 & $\begin{array}{l}\text { Keine relevante Beeinträchtigung. Kann trotz gewisser Symptome } \\
\text { Alltagsaktivitäten verrichten. }\end{array}$ \\
\hline 2 & $\begin{array}{l}\text { Leichte Beeinträchtigung. Kann sich ohne Hilfe versorgen, ist aber im } \\
\text { ohne Hilfe gehen. }\end{array}$ \\
\hline 3 & $\begin{array}{l}\text { Höhergradige Beeinträchtigung. Benötigt Hilfe bei der Körperpflege, } \\
\text { kann nicht ohne Hilfe gehen. }\end{array}$ \\
\hline 5 & $\begin{array}{l}\text { Schwere Behinderung. Bettlägerig, inkontinent, benötigt ständige } \\
\text { pflegerische Hilfe. }\end{array}$ \\
\hline 4
\end{tabular}

Modifizierte Rankin-Skala, mRS zur Beurteilung des Outcome des Patienten nach der Therapie.

Die Follow-up-mRS-Werten entsprechen den Werten in der klinischen Untersuchung 90 Tage nach dem akuten Ereignis. In Fällen von fehlenden Follow-up-mRS-Werten wurden stattdessen die Entlassung-mRS-Werte benutzt. Diese Parameter wurden mit dem Welcht-test in Fällen einer Normalverteilung (normal distribution) und mit dem Mann-Whitney- 
U-Test in Fällen einer nicht Normalverteilung (non-normal distribution) oder in Fällen einer ordinalen Distribution-Verteilung (ordinal distribution) analysiert. Kategorische Variablen zwischen beiden Gruppen wurden mit Fischer-exact-Test verglichen. Zusätzlich wurde die Probabilität eines guten klinischen Ergebnisses mit schrittweiser logistischer Regression (stepwise logistic regression) unter Verwendung aller in univarianter Analyse (univariate analysis) signifikant unterschiedlichen Variablen zwischen beiden Gruppen bewertet. Die Interobserver-Reproduzierbarkeit (Urteilerübereinstimmung - interobserver agreement) wurde mit Intra-Klassen-Korrelation (IKK-interclass correlation coefficient, ICC) analysiert. Die Analysen wurden mit dem MedCalc-Statistischen Paket (MedCalc, Mariakerke, Belgien) angefertigt. Das signifikante Niveau für alle Variablen war a=0.05. 


\section{Ergebnisse}

Der mediane NCCT-ASPECTS des Patientenkollektivs betrug 9 (Interquartilsabstand_Interquartile Range-IQR, 8 - 9), der mediane CTA-ASPECTS war 7 (IQR, 7 - 8) und der mediane CBV-ASPECTS betrug 8 (IQR, 6 - 8). Der mediane CBFASPECTS war 4 (IQR, 3-5) und der mediane TTD- und MTT-ASPECTS betrug 2 (IQR, $1-3)$ bzw. 3 (IQR, 1,75 - 3). Der mediane GSS-Score betrug -0,05 (IQR, -2,6 - 1,65). In Bezug auf die in der Behandlung maßgeblichen Zeitintervalle haben sich die folgenden Werte ergeben: Die mediane Zeit vom Symptombeginn bis zur Reperfusion betrug 254 Minuten (IQR, 222 - 317) und die mediane Zeit von der Leistenpunktion bis zur Reperfusion war 51 Minuten (IQR, 35 - 70).

Die Tabelle 4 fasst die Basis-Charakteristika, die Behandlungszeiten, die Scores der verschiedenen Parameter der Bildgebung und die klinischen Scores/Ergebnisse bei der Aufnahme, der Entlassung und der Untersuchung 90 Tagen nach dem Ereignis zusammen. Alle Parameter werden jeweils für das gesamte Patientenkollektiv sowie getrennt für Patienten mit gutem und mit schlechtem Outcome ausgewiesen.

Von 72 identifizierten Patienten wurden 7 Patienten aufgrund inkompletter Bildgebung ausgeschlossen. Fünfundsechzig Patienten wurden eingeschlossen, davon waren 26 Frauen. Das mediane Alter der Patienten betrug 72 J. (IQR, 68 - 76). Der mediane Aufnahme-NIHSS war 17 (IQR, 12 - 21) und der mediane Aufnahme-mRS war 5 (IQR, 4 -5). Bei allen Patienten wurde eine erfolgreiche Thrombektomie mit mTICI $2 b$ oder 3 in den finalen Angiographieserien durchgeführt. Bei 56\% der Fälle wurde die mechanische Thrombektomie mit intravenöser und intraarterieller Gabe von rt-PA kombiniert. In $26 \%$ 
der Fälle wurde eine mechanische Thrombektomie mit intravenöser rt-PA Gabe kombiniert und in den restlichen 12 Fällen wurde nur eine mechanische Thrombektomie durchgeführt. Der mediane Entlassungs-NIHSS war 7 (IQR, 4 - 12) und der mediane mRS bei Entlassung betrug 4 (IQR, 2 - 5). Der mediane Follow-up-mRS war 3 (IQR, 2 - 4). Insgesamt 40\% der Patienten wiesen im Follow-up ein gutes klinisches Ergebnis $(\mathrm{mRS} \leq 2)$ auf.

Von den analysierten Komorbiditäten (Begleiterkrankungen) waren nur Diabetes mellitus $(\mathrm{p}=0,013)$ und Adipositas $(\mathrm{p}=0,04)$ signifikant unterschiedlich zwischen den Patienten mit gutem und den Patienten mit schlechtem klinischen Ergebnis. Hinsichtlich der bildgebenden Variablen war der CBV-ASPECTS und die diversen Mismatch-Scores deutlich signifikant, $\mathrm{p}=0,0014$ bzw. $\mathrm{p}<0.01$. Sowohl der NCCT-ASPECTS als auch der GSS waren ebenfalls signifikant unterschiedlich, $\mathrm{p}=0,03$ bzw. $\mathrm{p}<0,0001$. Hinsichtlich der klinischen Scores war der Aufnahme-NIHSS und der Aufnahme-mRS mit einem p-Wert von 0,0003 bzw. $\mathrm{p}<0,0001$ zwischen den Gruppen signifikant unterschiedlich.

Die Patienten mit gutem klinischen Ergebnis wiesen einen medianen CTA-ASPECTS von 8 (IQR, 7 - 9) auf, während die Patienten mit schlechtem Ergebnis einen medianen CTAASPECTS von 7 (IQR, $7-8, \mathrm{p}=0,18$ ) aufwiesen. Die Zeitintervalle symptom onset to successful reperfusion (Symptombeginn bis zur Reperfusion) und groin puncture to successful reperfusion (Leistenpunktion bis zur Reperfusion) unterschieden sich nicht signifikant zwischen beiden Gruppen ( $p=0,16$ bzw. $p=0,91)$ (Tabelle 4). Die mediane Zeit vom Symptombeginn bis zur Rekanalisation betrug bei den Patienten mit gutem klinischen Ergebnis 237 Minuten (IQR, 205 - 306) und bei der Gruppe mit schlechtem klinischen Ergebnis 261 Minuten (IQR, 235 - 319). Das mediane Zeitintervall von der Leistenpunktion bis zur Reperfusion war 49 Minuten (IQR, 35 - 77) bzw. 51 Minuten 
(IQR, 33 - 69). Es zeigte sich kein statistisch signifikanter Unterschied in diesen Zeitintervallen.

Die Patientengruppe mit einem guten klinischen Ergebnis 90 Tage nach Symptombeginn wies einen medianen $\Delta(\mathrm{CBV}-\mathrm{CBF})-\mathrm{ASPECTS}$ von $4(\mathrm{IQR}, 3-5)$, einen medianen $\Delta(\mathrm{CBV}-\mathrm{TTD})$-ASPECTS von $6(\mathrm{IQR}, 5-7)$ und einen $\Delta(\mathrm{CBV}-\mathrm{MTT})$-ASPECTS von 5,5 (IQR, 4 - 6) auf. Die entsprechenden Werten der Gruppe mit mRS > 2 betrugen 3 (IQR, 1 $-3), 4(3-6)$ und $4(3-5)$.

Um den prognostischen Wert der bildgebenden Variablen zu untersuchen wurden die Untersucher gebeten, die sinnlosen Rekanalisationen vorauszubestimmen. Es handelt sich um Fälle mit erfolgreicher Thrombektomie des verschlossenen Gefäßes und mRS > 2 in der klinischen Untersuchung 90 Tage nach dem Ereignis. In Anbetracht der nur auf den NCCT- und CTA-Bildgebung basierten Parametern konnten die Rater 56\% der sinnlosen Rekanalisationen (Patienten mit erfolgreicher Eröffnung des Verschlusses und schlechtem klinischem Follow-up-Ergebnis) prognostizieren. Basierend auf dem CBV-ASPECTS konnten 96\% der sinnlosen Rekanalisationen korrekt vorhergesagt werden (Tabelle 5). Weiterhin sollten sie auf Basis der oben genannten Parameter auch die sinnvollen Rekanalisationen voraussagen (erfolgreiche Thrombektomie und finaler mRS $\leq 2$ ). Auf Basis des CTA-ASPECTS konnten nur 44\% korrekt identifiziert werden, während es im Falle des CBV-ASPECTS 61\% waren. Auf Basis des NCCT-ASPECTS konnten 100\% der sinnlosen, aber nur $43 \%$ der sinnvollen Thrombektomien vorausgesagt werden.

Tab. 4: Baseline-Charakteristika, Behandlungszeiten und klinisches Ergebnis zwischen Patienten mit gutem und schlechtem Follow-Up-Ergebnis 


\begin{tabular}{|c|c|c|c|c|}
\hline & $\begin{array}{c}\text { Insgesamt } \\
(\mathrm{n}=65)\end{array}$ & $\begin{array}{c}\text { Follow-up mRS } \\
\leq 2(\mathrm{n}=26)\end{array}$ & $\begin{array}{c}\text { Follow-up mRS } \\
>2(n=39)\end{array}$ & $P$ \\
\hline Alter, median (IQR), J & $72(68-76)$ & $71,5(59-75)$ & $73(69-78)$ & 0,11 \\
\hline $\begin{array}{l}\text { Aufnahme NIHSS, } \\
\text { median (IQR) }\end{array}$ & $17(12-21)$ & $14(10-17)$ & $18(15-24)$ & $0,0003^{*}$ \\
\hline $\begin{array}{l}\text { Aufnahme mRS, median } \\
\text { (IQR) }\end{array}$ & $5(4-5)$ & $4(4-5)$ & $5(5-5)$ & $<0,0001^{*}$ \\
\hline \multicolumn{5}{|l|}{ Komorbitäten } \\
\hline Hyperlipidämie & $42(65 \%)$ & $17(65 \%)$ & $25(64 \%)$ & 1 \\
\hline Arterielle Hypertonie & $57(88 \%)$ & $21(81 \%)$ & $36(92 \%)$ & 0,25 \\
\hline Diabetes mellitus & $19(30 \%)$ & $3(12 \%)$ & $16(41 \%)$ & $0,013^{*}$ \\
\hline Rauchen & $18(30 \%)$ & $7(30 \%)$ & $11(30 \%)$ & 1 \\
\hline PAD & $2(3 \%)$ & $1(4 \%)$ & $1(3 \%)$ & 1 \\
\hline Adipositas & $33(55 \%)$ & $9(38 \%)$ & $24(67 \%)$ & $0,04 *$ \\
\hline \multicolumn{5}{|l|}{ Scores, median (IQR) } \\
\hline CT ASPECTS & $9(8-9)$ & $9(9-9)$ & $8(7-9)$ & $0,03 *$ \\
\hline CTA ASPECTS & $7(7-8)$ & $8(7-9)$ & $7(7-8)$ & 0,18 \\
\hline CBV ASPECTS & $8(6-8)$ & $8(8-9)$ & $6(5-8)$ & $0,0001^{*}$ \\
\hline CBF ASPECTS & $4(3-5)$ & $4(4-5)$ & $4(3-5)$ & 0,27 \\
\hline TTD ASPECTS & $2(1-3)$ & $2.5(1-3)$ & $2(1-3)$ & 0,53 \\
\hline MTT ASPECTS & $3(1.75-3)$ & $3(2-3)$ & $3(1-3)$ & 0,62 \\
\hline$\Delta(\mathrm{CBV}-\mathrm{CBF}) \mathrm{ASPECTS}$ & $3(1-4)$ & $4(3-5)$ & $3(1-3)$ & $0,0014^{*}$ \\
\hline$\Delta(\mathrm{CBV}-\mathrm{TTD}) \mathrm{ASPECTS}$ & $5(4-6)$ & $6(5-7)$ & $4(3-6)$ & $0,0012^{*}$ \\
\hline$\Delta(\mathrm{CBV}-\mathrm{MTT}) \mathrm{ASPECTS}$ & $4(3-6)$ & $5.5(4-6)$ & $4(3-5)$ & $0,0008 *$ \\
\hline GSS & $\begin{array}{c}-0.05(-2.6- \\
1.65)\end{array}$ & $1.6(0.25-2.4)$ & $\begin{array}{c}-1.5(-3.6-- \\
0.01)\end{array}$ & $<0,0001^{*}$ \\
\hline $\begin{array}{l}\text { Symptombeginn bis } \\
\text { mTICI2b, median (IQR) }\end{array}$ & $\begin{array}{l}254(222- \\
317)\end{array}$ & $237(205-306)$ & $261(235-319)$ & 0,16 \\
\hline $\begin{array}{l}\text { Leistenpunktion bis } \\
\text { mTICI2b, median (IQR) }\end{array}$ & $51(35-70)$ & $49(35-77)$ & $51(33-69)$ & 0,91 \\
\hline $\begin{array}{l}\text { Entlassung-NIHSS, } \\
\text { median (IQR) }\end{array}$ & $7(4-15)$ & $3(1-5)$ & $13(9-20)$ & $<0,0001^{*}$ \\
\hline $\begin{array}{l}\text { Entlassung-mRS, median } \\
\text { (IQR) }\end{array}$ & $4(2-5)$ & $1.5(1-2)$ & $5(4-5)$ & $<0,0001^{*}$ \\
\hline $\begin{array}{l}\text { Follow-up mRS, median } \\
\text { (IQR) }\end{array}$ & $3(2-4)$ & $1(1-2)$ & $4(4-5)$ & $<0,0001^{*}$ \\
\hline
\end{tabular}


mRS, modifizierter Rankin Score; IQR, interquartile range; NIHSS, National Institutes of Health stroke scale; PAD, peripheral artery disease (periphere arterielle Verschlusskrankheit); ASPECTS, Alberta stroke program early CT scale; CTA, CT angiography; CBV, cerebral blood volume; CBF, cerebral blood flow; TTD, time to drain; MTT, mean transit time; GSS, Göttingen stroke scale; TICI, thrombolysis in cerebral infarction. Daten aus Tsogkas et al. (2016), mit freundlicher Genehmigung von Springer Nature, licence number:4923090199089 (entspricht Tabelle 1).

*P value significant

Tab. 5: Retrospektive Prädiktion des klinischen Ergebnisses basierend auf AufnahmeNCCT-ASPECTS, /-CTA-ASPECTS oder/-CBV-ASPECTS

\begin{tabular}{|c|c|c|c|c|}
\hline & $\begin{array}{l}\text { Raters' } \\
\text { Meinung }\end{array}$ & $\begin{array}{c}\text { Follow-up- } \\
\text { mRS } \leq 2\end{array}$ & $\begin{array}{c}\text { Follow-up- } \\
\text { mRS > }\end{array}$ & $\begin{array}{c}\text { Fisher's exact } \\
\text { Test } P \text { value }\end{array}$ \\
\hline \multirow{2}{*}{$\begin{array}{l}\text { Entscheidung } \\
\text { anhand des } \\
\text { NCCT- } \\
\text { ASPECTS }\end{array}$} & $\begin{array}{l}\text { Sinnlose } \\
\text { Reperfusion }\end{array}$ & $0 \%$ & $100 \%$ & \multirow{2}{*}{0,07} \\
\hline & $\begin{array}{l}\text { Sinnvolle } \\
\text { Reperfusion }\end{array}$ & $43 \%$ & $57 \%$ & \\
\hline \multirow{2}{*}{$\begin{array}{l}\text { Entscheidung } \\
\text { anhand des- } \\
\text { CTA- } \\
\text { ASPECTS }\end{array}$} & $\begin{array}{l}\text { Sinnlose } \\
\text { Reperfusion }\end{array}$ & $44 \%$ & $56 \%$ & \multirow{2}{*}{0,77} \\
\hline & $\begin{array}{l}\text { Sinnvolle } \\
\text { Reperfusion }\end{array}$ & $39 \%$ & $61 \%$ & \\
\hline \multirow{2}{*}{$\begin{array}{l}\text { Entscheidung } \\
\text { anhand des } \\
\text { CBV- } \\
\text { ASPECTS }\end{array}$} & $\begin{array}{l}\text { Sinnlose } \\
\text { Reperfusion }\end{array}$ & $4 \%$ & $96 \%$ & \multirow{2}{*}{$<0,0001$} \\
\hline & $\begin{array}{l}\text { Sinnvolle } \\
\text { Reperfusion }\end{array}$ & $61 \%$ & $39 \%$ & \\
\hline
\end{tabular}

NCCT, noncontrast CT; CTA, CT angiography; ASPECTS, Alberta stroke program early CT scale; CBV, cerebral blood volume; mRS, modified Rankin score. Daten aus Tsogkas et al. (2016), mit freundlicher Genehmigung von Springer Nature, licence number:4923090199089 (entspricht Tabelle 2).

Die receiver operating characteristic analysis (ROC-Kurvenanalyse) zeigte ähnliche Ergebnisse. Sensitivität, Spezifität, positiver prädiktiver und negativer Wert (PPW, NPW) für NCCT-ASPECTS < 7 war 8\%, 100\%, 100\% und 42\%. Für CTA-ASPECTS < 7 
betrugen die Werte 23\%, 77\%, 60\% und 40\%. Für CBV-ASPECTS zeigt sich ein optimal criterion value von $<7$ mit Sensitivität von $49 \%$, Spezifität von $96 \%$, PPW von $95 \%$ und NPW von $56 \%$ (Abb. 6).

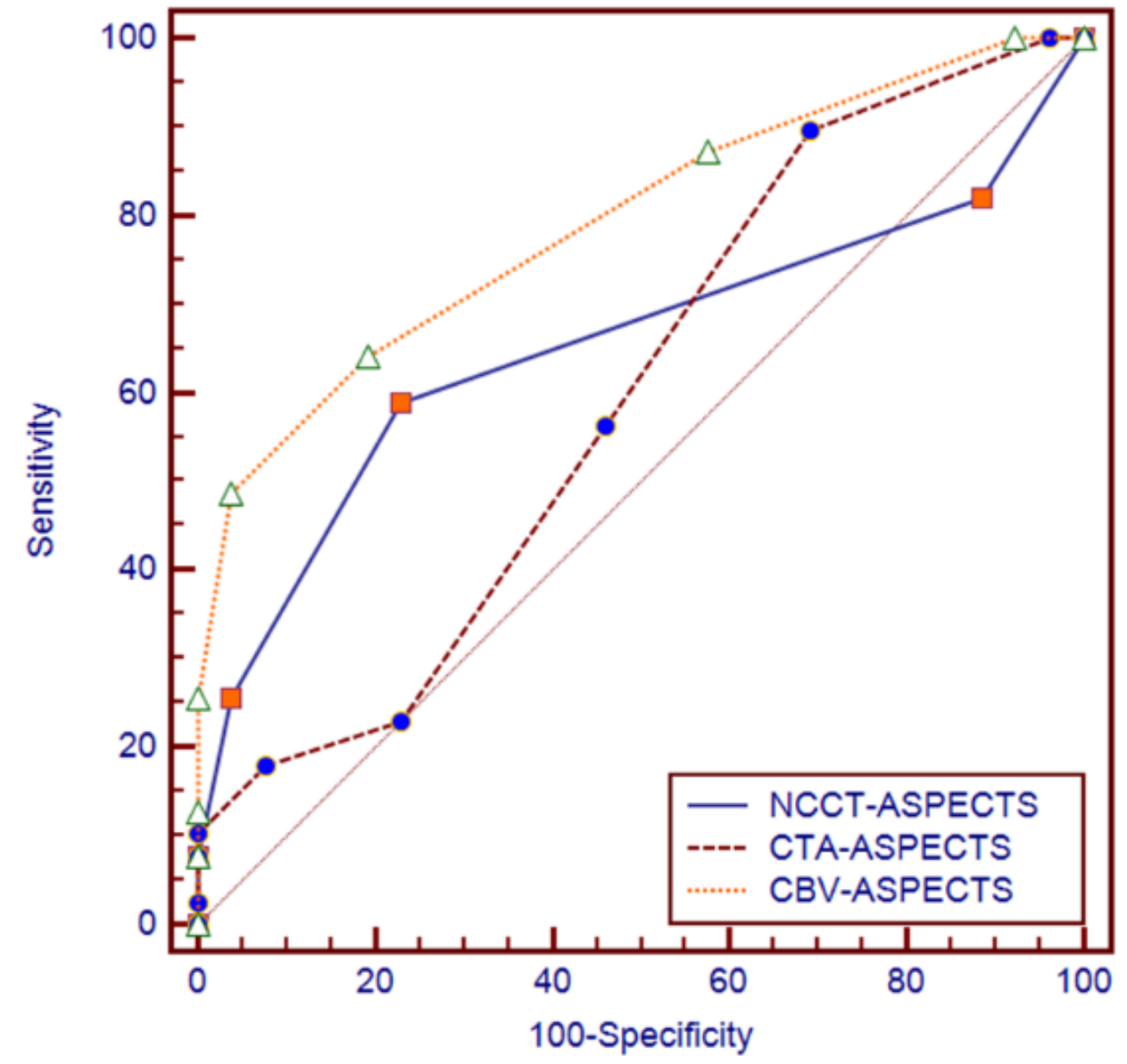

Abb. 6: Vergleich der ROC-Kurvenanalysen von NCCT-ASPECTS, CTA-ASPECTS und CBVASPECTS. Daten aus Tsogkas et al. (2016), mit freundlicher Genehmigung von Springer Nature, licence number:4923090199089 (entspricht Abbildung 2).

Laut schrittweiser logistischer Regression waren nur der Aufnahme-NIHSS ( $p=0,04 ;$ odds ratio 1,14; $95 \%$ confidence interval $[\mathrm{CI}] 1,00$ bis 1,31$)$ und der CBV-ASPECTS ( $p=0,006$; odds ratio 0,$45 ; 95 \%$ CI 0,26 bis 0,79 ) signifikante Beitragende (contributors) zur 
Prädiktion des klinischen Ergebnisses. Die Urteilerübereinstimmung zwischen den drei Rater war sehr gut mit IKK-Werten von 0,92 für CBV-ASPECTS (95\% CI, 0,89-0,95), 0,9 für CBF-ASPECTS (95\% CI, 0,85 - 0,93), 0,7 für NCCT-ASPECTS (95\% CI, 0,59 0,79), 0,88 für CTA-ASPECTS (95\% CI, 0,82 - 0,92), 0,71 für MTT-ASPECTS (95\% CI, $0,6-0,8)$ und 0,85 für TTD-ASPECTS $(95 \% \mathrm{CI}, 0,78-0,9)$. 


\section{Diskussion}

Die endovaskuläre Behandlung der mit Blutgerinnsel verschlossenen hirnversorgenden Arterie (Neuro-Thrombektomie) ist seit mehr als zwei Jahrzehnten technisch möglich. Trotzdem war allein die systemische Thrombolyse mittels intravenös verabreichtem rt-PA bis 2015 die Standardtherapie des akuten ischämischen Schlaganfalls für Patienten, die innerhalb von 4,5 Stunden nach Symptombeginn behandelt werden konnten. Die im Jahr 2013 publizierten Studien IMS III, SYNTHESIS und MR RESCUE (Broderick et al. 2013; Ciccone et al. 2013; Kidwell et al. 2013) verglichen die Kombination aus endovaskulärer Behandlung und medikamentöser Therapie mit der alleinigen medikamentösen Therapie bei Patienten mit akutem ischämischen Schlaganfall im Zeitfenster von 4,5 Stunden nach dem Ereignis. Keine der Studien konnte einen signifikanten Effekt auf das klinische Outcome der Patienten durch die zusätzliche Thrombektomie 90 Tage nach Therapie zeigen. Weil die Thrombektomie ein teures und ressourcenintensives Verfahren war und bis zur Entwicklung der neuen Devices mit relativ häufigen periprozeduralen Komplikationen einherging, wurde sie bis 2015 nur in ausgewählten Einzelfällen und hoch spezialisierten Zentren durchgeführt.

In einer Subgruppenanlyse dieser Studien war jedoch zu erkennen, dass die verzögerte Behandlung, eine nicht optimale Patientenselektion und die erfolglose endovaskuläre Behandlung wahrscheinlich für den fehlende Nutzen der kombinierten Therapie verantwortlich waren. Darüber hinaus wurden Patienten ohne Verschluss einer proximalen Arterie eingeschlossen. Diese Interpretation wird von einer 2012 publizierten Studie unterstützt. Diese konnte in Patienten, die in der multimodalen Bildgebung eine große 
Penumbra aufwiesen zeigen, dass eine schnelle Reperfusion mittels Thrombektomie das klinische Ergebnis signifikant verbessert (Lansberg et al. 2012).

Die Reperfusion des betroffenen Gefäßes ist eine Hauptvoraussetzung für den Therapieerfolg. Die oben erwähnten Erkenntnisse deuten allerdings darauf hin, dass die Rekanalisation allein nicht ausreicht. Es ist essentiell, durch eine richtige Patientenselektion die beste Therapieoption individuell zu ermitteln. Die multimodale Bildgebung und vor allem die CT-Perfusion waren für die Selektion der Patienten, die endovaskulär behandelt werden sollten von großer Bedeutung. Die Erkennung der Prädiktoren für eine sinnvolle oder sinnlose Thrombektomie war der Hintergrund mehrerer Studien. Diese Prädiktoren wurden während der Entscheidung für oder gegen Thrombektomie im klinischen Alltag häufig verwendet.

Unsere Ergebnisse bestätigen früher publizierte Studien, die die Verwendung des CBVASPECTS als Biomarker in der Therapie des akuten ischämischen Schlaganfalls vorschlagen (Aviv et al. 2007; Kim et al. 2010). Die univariante Analyse und die logistische Regression deuten darauf hin, dass der CBV-ASPECTS ein signifikanter Prädiktor des klinischen Ergebnisses der Patienten war, die nach akutem ischämischen Schlaganfall erfolgreich durch eine Thrombektomie behandelt wurden. Der p-Wert von CBV-ASPECTS war 0,006 und das Quotenverhältnis (odds ratio-OR) 0,45.

Unter Verwendung des CBV-ASPECTS konnten die Rater 96\% der sinnlosen Rekanalisationen (erfolgreiche Thrombektomie und schlechtes klinisches Ergebnis) prognostizieren. Ein anderer signifikanter Prädiktor war der Aufnahme-NIHSS. Unsere Ergebnisse widersprechen der Arbeit von Lum et al., dass der CTA-ASPECTS ein geeigneter Prädiktor des klinischen Ergebnisses nach erfolgreicher Thrombektomie ist 
(Lum et al. 2014) (Abb. 7). In dieser Studie wurden die CTA-Bilddaten von der CTPerfusion unter Verwendung eines prädefinierten Werts der Kontrast-Zeit-Kurve extrahiert. In unserer Studie benutzten wir ein single-phase-Protokoll für die CTA mit Bolus-adaptiertem Delay (Verzögerung). Dieses Protokoll ist ein häufig verwendetes Protokoll für die Erkennung oder den Ausschluss einer Einengung oder eines Verschlusses der hirnversorgenden extrakraniellen und intrakraniellen Arterien. In einer Studie mit älteren CT-Geräten zeigte sich kein signifikanter Unterschied bezüglich der Läsionslast zwischen den CBV-Bildern und den CTA-Bildern (Schramm et al. 2004). Unter Verwendung der neuen deutlich schnelleren Geräte (fast multisection scanners) haben Studien gezeigt, dass die CTA-Bilder eher CBF- als CBV-gewichtet sind, daher wird der Infarktkern überschätzt (Sharma et al. 2011). Unsere Ergebnisse stimmten mit dieser Interpretation überein, da der CTA-ASPECTS kein statistisch signifikanter Prädiktor des klinischen postinterventionellen Ergebnisses in der logistischen Regression war. In Anbetracht der CTA-Bildgebung konnten die Rater nur 56\% der sinnlosen Rekanalisationen prädizieren. 


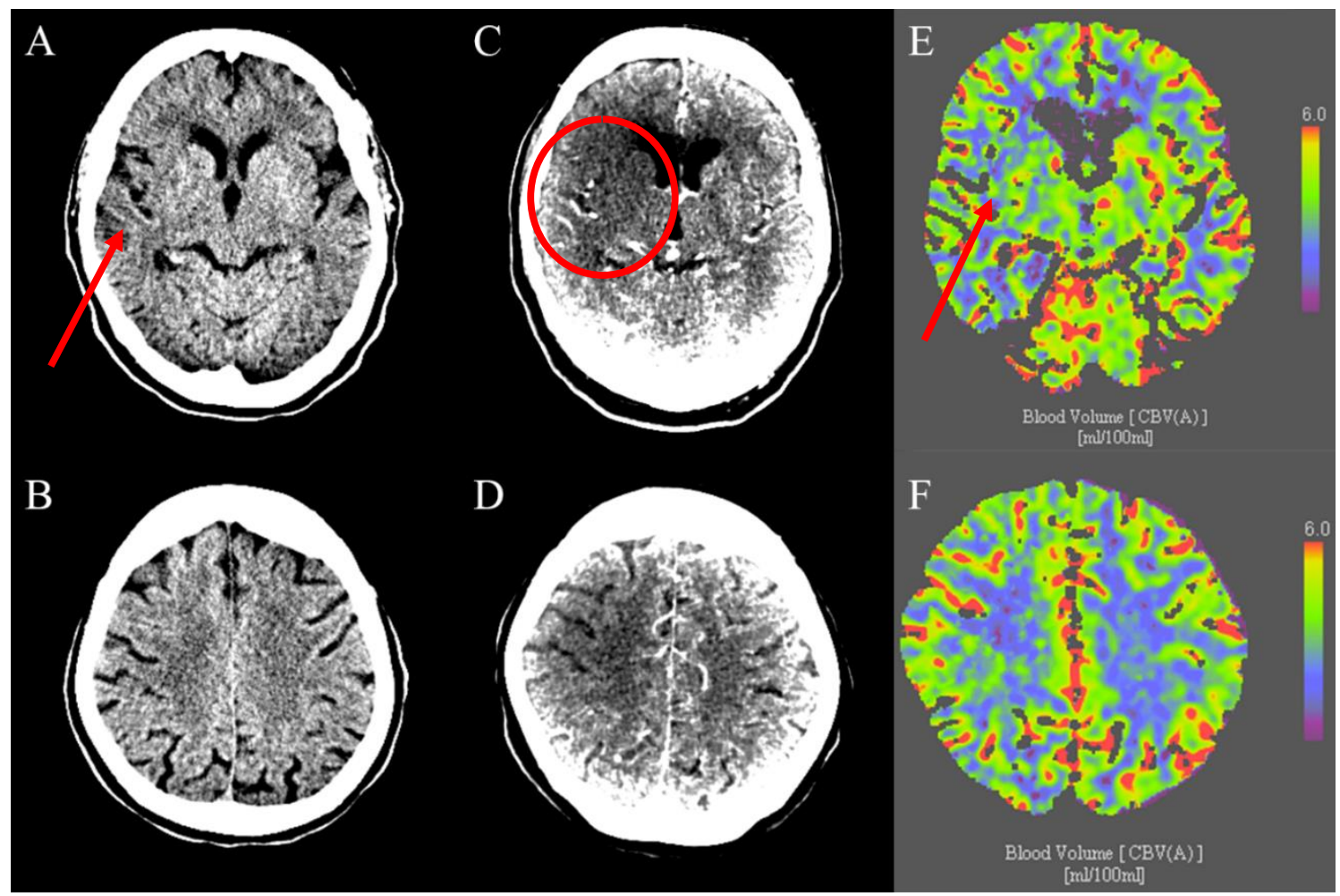

Abb. 7: Bilder einer 75-jährigen Patientin mit Aufnahme-NIHSS von 16 und Aufnahme-mRS von 5. Das NCCT (A, B) zeigt Frühzeichen eines Infarktes nur im rechten insulären Kortex, somit NCCT-ASPECTS von 9. Die CTA-Bilder (C, D) weisen Hypodensitäten im rechten Caput nuclei caudati, im Linsenkern, im insulären Kortex und im frontalen Operculum auf, einem CTAASPECTS von 6 entsprechend. Die CBV-Karten (E, F) zeigen eine Minderung des zerebralen Blutvolumens nur im rechten insulären Kortex, CBV-ASPECTS 9. Nach erfolgreicher endovaskulärer Thrombektomie des M1-Verschlusses (Zeit von Leistenpunktion bis zur Rekanalisation [TICI 2b] 45 Minuten) wies die Patientin eine signifikante Verbesserung der Symptome auf und wurde mit mRS von 1 entlassen. Daten aus Tsogkas et al. (2016), mit freundlicher Genehmigung von Springer Nature, licence number: 4923090199089 (entspricht Abbildung 3).

Die fünf oben genannten randomisierten Studien, die 2015 publiziert worden sind, zeigten eine deutliche Überlegenheit der mit endovaskulärer Behandlung kombinierten Therapie des Schlaganfalls im Vergleich zur (isolierten) IV Lyse bei Patienten mit Verschluss eines proximalen Gefäßes (z.B. der Arterie cerebri media) (Campbell und Mitchell 2015; 
Fransen et al. 2014; Goyal et al. 2015; Jovin et al. 2015; Saver et al. 2015). Die Quote der Patienten mit gutem klinischen Ergebniss nach endovaskulärer Therapie variierten von $33 \%$ (MR-CLEAN) bis 71\% (EXTEND-IA). Die Studie mit den prozentuell geringsten guten klinischen Ergebnissen $(m R S \leq 2)$ hatte die breitesten Einschlusskriterien und benötigte keinen Nachweis eines kleinen Infarktkerns in der präinterventionellen Bildgebung. Im Gegensatz dazu war die CT-Perfusion in der Studie mit den besten Ergebnissen für die Patientenselektion notwendig.

Die Verwendung des CBV-ASPECTS als eines von den Kriterien zur Patientenselektion führte in unserem Institut zu einem deutlichen Anstieg der guten Ergebnisse in den Jahren 2012-2014. Von 2007 bis 2010 waren ca. 34\% aller erfolgreichen Rekanalisationen mit einem guten klinischen Ergebnis verbunden, verglichen zu ca. 60\% von 2012 bis 2014. In den Jahren 2012 bis 2014 wurde ein CBV-ASPECTS von mindestens 8 als Entscheidungskriterium für die mechanische Thrombektomie genutzt. In den vorherigen Jahren (2007 bis 2010) reichte ein CBV-ASPECTS von mindestens 6 aus.

Nach der Publikation der oben genannten Studien (2015) wird der CBV-ASPECTS als Entscheidungskriterium bei Patienten mit Symptombeginn innerhalb der ersten 6 Stunden nicht mehr verwendet. Eine weitere Verwendung ist ethisch nicht vertretbar in Anbetracht der Ergebnisse einer Metaanalyse der oben genannten Studien, die 2016 publiziert worden ist (Goyal et al. 2016). Insgesamt wurden 1287 Patienten (634 in der endovaskulären Interventionsgruppe und 653 in der Kontrollgruppe) analysiert. Es zeigte sich eindeutig ein positiver Effekt der mechanischen Thrombektomie bei Patienten mit akutem ischämischen Schlaganfall im frühen Zeitfenster (bis zu sechs Stunden nach Symptombeginn) auf das klinische Ergebnis nach 90 Tagen ( $\mathrm{p}<0,0001)$, auch wenn keine erweiterte Bildgebung 
(Perfusion) durchgeführt wurde. Hierbei zeigte sich insbesondere in der Subgruppenanalyse, dass der Behandlungseffekt auch bei bereits großen Infarkten (entsprechend einem niedrigen ASPECTS in der initialen Bildgebung) erhalten bleibt. Dies deckt sich mit Ergebnissen aus der MR CLEAN Studie, die einen positiven Behandlungseffekt gezeigt hat, ohne weitergehende bildgebende Parameter, insbesondere ohne Einbeziehung der Perfusionsbildgebung (Berkhemer et al. 2015).

Infolge der oben genannten Evidenz verwenden wir den CBV-ASPECTS nur noch zur Selektion von Schlaganfallpatienten bei denen der Symptombeginn entweder länger als sechs Stunden zurückliegt oder nicht eruiert werden kann (Wake-Up-Stroke). Das Hauptargument gegen eine zu enge Selektion in der Behandlung des akuten Schlaganfalls ist die Reduzierung der Anzahl der Patienten, die mit der effektivsten Therapie behandelt werden. Nicht behandelte Patienten könnten immer noch von der Thrombektomie profitieren. Ca. 30\% der Patienten mit Verschluss eines proximalen Gefäßes, die in die NCCT-basierten Studien eingeschlossen geworden wären, wurden in die EXTEND-IA nicht eingeschlossen. Dies könnte den Wert einer engen Selektion theoretisch reduzieren und diesbezüglich, in Anbetracht der Shift-Analyse, wird eine konsistente Verbesserung der Ergebnisse aller Patientengruppe nachgewiesen. Verglichen zur Verwendung nur der intravenösen Therapie, deutet es auf einen statistischen Vorteil der Kombination der endovaskulären Therapie mit der IV Lyse (wenn indiziert) für alle Patienten hin.

In unserer Studie war der einzige Patient mit CBV-ASPECTS $<7$ und gutem klinischem Ergebnis sehr jung (27 Jahren alt) und hatte einen moderaten Aufnahme-NIHSS-Score (10 Punkte). Aus diesem Grund wurde von uns ein neuer Score entwickelt, der GSS (Göttinger Stroke Score). Dieser kombiniert den Aufnahme-NIHSS, den CBV-ASPECTS sowie das 
Alter. In unserem Kollektiv war der GSS statistisch signifikant unterschiedlich $(\mathrm{p}<0,0001)$ zwischen beiden Gruppen mit einem Median von 1,6 (IQR 0,25 - 2,4) bzw. -1,5 (IQR -3,6 - -0,01) (Tabelle 4). Trotz der positiven Ergebnisse soll der GSS vor seiner klinischen Verwendung weiter untersucht und validiert werden.

Gemäß den aktuellen Leitlinien (Deutsche Gesellschaft Neurologie-Leitlinien für Diagnostik und Therapie in der Neurologie, Akuttherapie des ischämischen SchlaganfallsErgänzung 2015) soll die Thrombektomie bei allen Patienten mit akutem ischämischen Schlaganfall durch einen großen arteriellen Verschluss der vorderen Zirkulation bis zu 6 Stunden nach Symptombeginn durchgeführt werden. Im späteren Zeitfenster $(>6$ Stunden oder Wake-Up-Stroke) kann die Thrombektomie als Therapie ebenfalls in Betracht gezogen werden, sofern sich in der erweiterten Bildgebung noch rettbares Hirngewebe zeigt. Hierbei handelt es sich um eine Einzelfallentscheidung, die auf Basis der CTPerfusion oder einer multiphasischen CT-Angiographie getroffen werden sollte.

2018 wurden zwei randomisiert-kontrollieren Untersuchungen zur Wirkung der mechanischen Thrombektomie bei Patienten mit einem großen Gefäßverschluss der vorderen Zirkulation im Zeitfenster von 6 bis 24 Stunden oder im Falle eines Wake-UpStrokes publiziert, die DAWN und DEFUSE III Studien (Albers et al. 2018; Nogueira et al. 2018). Beide Studien haben ein Missmatch-Konzept zur Patientenselektion genutzt. In der DAWN Studie wurde ein Missmatch zwischen Infarktkern und klinischer Präsentation als Haupteinschlusskriterium genutzt. So wurde z.B. bei Patienten mit einem Alter von unter 80 Jahren ein NIHSS $\geq 10$ und ein Infarktkern $<31 \mathrm{~mL}$ bzw. ein NIHSS $\geq 20$ und ein Infarktkern $<51 \mathrm{~mL}$ als Hauptkriterium genutzt. Der Infarktkern wurde dabei automatisiert entweder in der DWI-Sequenz (MRT) oder der CT-Perfusion (rCBF) 
bestimmt. Die Theorie war, dass der ermittelte Infarktkern das Defizit nicht erklären konnte und somit noch rettbares Gewebe (Penumbra) vorhanden sei. In der Interventionsgruppe (Thrombektomie) zeigte sich ein signifikant besseres klinisches Ergebnis (49\% funktionell unabhängig vs $13 \%$ in der Kontrollgruppe). Es zeigte sich kein Unterschied in der Mortalität und der Prävalenz von intrakraniellen Blutungen. Die DEFUSE III Studie zeigte bei leicht anderen Einschlusskriterien ebenfalls eine eindeutige Überlegenheit der mechanischen Thrombektomie. In dieser Studie wurden Patienten im Zeitfenster von 6 bis 16 Stunden und im Alter von 18 bis 90 Jahre eingeschlossen. Der NIHSS Score musste mind. 6 betragen. Im Gegensatz zur DAWN Studie wurde aber das Missmatch rein bildmorphologisch definiert. Es wurden alle Patienten mit einem Infarktkern $<70 \mathrm{~mL}$ und einem Verhältnis von Penumbra zu Infarktkern (Penumbra Volume/Infarktvolume) $\geq 1.8$ eingeschlossen.

Die Ergebnisse beider Studien zeigen eindeutig, dass auch Patienten im erweiterten Zeitfenster von der Thrombektomie profitieren können. Hier scheint allerdings die Patientenselektion eine deutlich wichtigere Rolle zu spielen als im früheren Zeitfenster. Da allerdings die automatische Volumetrierung von Infarktkern und Penumbra das Vorhandensein von oftmals kostspieligen Programmen voraussetzt, ist die Entwicklung und Untersuchung von einfach zu bestimmenden Prädiktoren (wie der CBV-ASPECTS oder der GSS) für ein gutes klinisches post-therapeutisches Ergebnis von essentieller Bedeutung, um sinnlose Rekanalisationen zu vermeiden. In unserer Studie waren der CBVASPECTS und der GSS die besten Prädiktoren des klinischen Outcomes.

Die Hauptlimitationen unserer Studie sind der retrospektive Charakter der Analyse und die streng selektierten Patientengruppen, da nur Patienten mit erfolgreichen Rekanalisationen 
eingeschlossen wurden. Die Daten wurden aus unserer prospektiv erhobenen Datenbank extrahiert, jedoch wurden die CTP-Bilder nur im Zeitraum September 2012 bis Dezember 2014 zur Therapieentscheidung verwendet. Vor September 2012 war das native Computertomogramm das einzige Auswahlkriterium zur endovaskulären Behandlung. Das oben beschriebene Modell zur Therapieentscheidung erklärt das Paradox, dass die Zeit (entweder Symptombeginn bis Rekanalisation oder Leistenpunktion bis Rekanalisation) in unserer Studie kein signifikanter Prädiktor des klinischen Ergebnisses war. Eine weitere Limitation ist, dass unsere Ergebnisse bei CTP-Daten nicht appliziert werden könnten, die mittels anderer Protokolle und Softwares akquiriert wurden. Dies Argument wurde in einer älteren Studie gezeigt (Fahmi et al. 2012). Darüber hinaus haben wir den prognostischen Wert der Kombination des CBV-ASPECTS mit dem CTA-ASPECTS nicht analysiert, da mehrere Studien die Überlegenheit der Kombination bereits konfirmiert haben (Lee et al. 2013). 


\section{Zusammenfassung}

Der Schlaganfall ist die dritthäufigste Todesursache und die häufigste Ursache für Langzeitbehinderung in Deutschland. Aktuell sind sowohl die systemische Thrombolyse (rt-PA) als auch die Thrombektomie die Therapie der Wahl für proximale Verschlüsse innerhalb von 4,5 Stunden. Von essenzieller Bedeutung sind die erfolgreiche Reperfusion der okkludierten Arterie und der rasche Ansatz der Therapie. Studien konnten beweisen, dass selektierte Patienten, auch nach sechs Stunden von der Thrombektomie profitieren können. Die Selektion soll mittels multimodaler Bildgebung durchgeführt werden.

Unsere Studie legt nahe, dass der CBV-ASPECTS ein signifikanter Prädiktor des klinischen Ergebnisses nach Thrombektomie bei Patienten mit akutem ischämischen Schlaganfall ist. Aufgrund seines niedrigen prognostischen Wertes raten wir von der Verwendung des einphasigen CTA-ASPECTS, akquiriert mit modernen schnellen CTScannern, als eines Biomarkers ab. Unsere Ergebnisse sollten nicht appliziert werden, wenn die CTA-Bilddaten von der CT-Perfusion extrahiert oder multiphasige CTAProtokolle verwendet werden. 


\section{Literaturverzeichnis}

Albers GW, Marks MP, Kemp S, Christensen S, Tsai JP, Ortega-Gutierrez S, McTaggart RA, Torbey MT, Kim-Tenser M, Leslie-Mazwi T et al. (2018): Thrombectomy for stroke at 6 to 16 hours with selection by perfusion imaging $\mathrm{N}$ Engl J Med $\underline{378}, 708-718$

Aviv RI, Mandelcorn J, Chakraborty S, Gladstone D, Malham S, Tomlinson G, Fox AJ, Symons S (2007): Alberta Stroke Program Early CT Scoring of CT perfusion in early stroke visualization and assessment AJNR Am J Neuroradiol 28, 1975-1980

Barber PA, Demchuk AM, Zhang J, Buchan AM (2000): Validity and reliability of a quantitative computed tomography score in predicting outcome of hyperacute stroke before thrombolytic therapy. ASPECTS Study Group. Alberta Stroke Programme Early CT Score Lancet $\underline{355}, 1670-1674$

Berkhemer OA, Fransen PS, Beumer D, van den Berg LA, Lingsma HF, Yoo AJ, Schonewille WJ, Vos JA, Nederkoorn PJ, Wermer MJ et al. (2015): A randomized trial of intraarterial treatment for acute ischemic stroke N Engl J Med $\underline{372}$, 11-20

Broderick JP, Palesch YY, Demchuk AM, Yeatts SD, Khatri P, Hill MD, Jauch EC, Jovin TG, Yan B, Silver FL et al. (2013): Endovascular therapy after intravenous t-PA versus t-PA alone for stroke N Engl J Med $\underline{368}$, 893-903

Campbell BC, Mitchell PJ (2015): Endovascular therapy for ischemic stroke N Engl J Med $\underline{372}, 2365-2366$

Campbell BC, Mitchell PJ, Kleinig TJ, Dewey HM, Churilov L, Yassi N, Yan B, Dowling RJ, Parsons MW, Oxley TJ et al. (2015): Endovascular therapy for ischemic stroke with perfusion-imaging selection N Engl J Med $\underline{372}$, 1009-1018

Ciccone A, Valvassori L, Nichelatti M, Sgoifo A, Ponzio M, Sterzi R, Boccardi E (2013): Endovascular treatment for acute ischemic stroke N Engl J Med $\underline{368}, 904-913$

Coutts SB, Demchuk AM, Barber PA, Hu WY, Simon JE, Buchan AM, Hill MD (2004):

Interobserver variation of ASPECTS in real time Stroke $\underline{35}$, e103-105

Destatis: https://www-genesis.destatis.de; (Suchbegriff : Schlaganfall, Todesraten)

Zugriff am 13.02.2018

DGN (2016): Akuttherapie des ischämischen Schlaganfalls - Ergänzung 2015.

Rekanalisierende Therapie - Leitlinienreport. Hrsg.: Kommission Leitlinien der 
Deutschen Gesellschaft für Neurologie

[https://www.awmf.org/uploads/tx_szleitlinien/030-

140m_S2k_akuter_ischaemischer_schlaganfall_2016-05.pdf]

Fahmi F, Marquering HA, Streekstra GJ, Beenen LF, Velthuis BK, VanBavel E, Majoie CB (2012): Differences in CT perfusion summary maps for patients with acute ischemic stroke generated by 2 software packages AJNR Am J Neuroradiol 33, 2074-2080

Fransen PS, Beumer D, Berkhemer OA, van den Berg LA, Lingsma H, van der Lugt A, van Zwam WH, van Oostenbrugge RJ, Roos YB, Majoie CB et al. (2014): MR CLEAN, a multicenter randomized clinical trial of endovascular treatment for acute ischemic stroke in the Netherlands: study protocol for a randomized controlled trial Trials $\underline{15}, 343$

Gonzalez RG (2006): Imaging-guided acute ischemic stroke therapy: From "time is brain" to "physiology is brain" AJNR Am J Neuroradiol 27, 728-735

Goyal M, Demchuk AM, Menon BK, Eesa M, Rempel JL, Thornton J, Roy D, Jovin TG, Willinsky RA, Sapkota BL et al. (2015): Randomized assessment of rapid endovascular treatment of ischemic stroke N Engl J Med 372, 1019-1030

Goyal M, Menon BK, van Zwam WH, Dippel DW, Mitchell PJ, Demchuk AM, Davalos A, Majoie CB, van der Lugt A, de Miquel MA et al. (2016): Endovascular thrombectomy after large-vessel ischaemic stroke: a meta-analysis of individual patient data from five randomised trials Lancet $\underline{387}$, 1723-1731

Hacke W, Kaste M, Bluhmki E, Brozman M, Davalos A, Guidetti D, Larrue V, Lees KR, Medeghri Z, Machnig T et al. (2008): Thrombolysis with alteplase 3 to 4.5 hours after acute ischemic stroke N Engl J Med 359, 1317-1329

Handschu R, Fateh-Moghadam S, Klotz E, Schmid A, Stemper B, Heckmann JG, Huk WJ, Neundorfer B, Tomandl BF (2004): Multimodale Computertomographie beim akuten Hirninfarkt. Erfahrungen bei einem standardisierten Untersuchungsprotokoll bei 100 Patienten. Nervenarzt $\underline{75}, 564-576$

Hill MD, Demchuk AM, Goyal M, Jovin TG, Foster LD, Tomsick TA, von Kummer R, Yeatts SD, Palesch YY, Broderick JP (2014): Alberta Stroke Program early computed tomography score to select patients for endovascular treatment: Interventional Management of Stroke (IMS)-III Trial Stroke 45, 444-449

Jovin TG, Chamorro A, Cobo E, de Miquel MA, Molina CA, Rovira A, San Roman L, Serena J, Abilleira S, Ribo M et al. (2015): Thrombectomy within 8 hours after symptom onset in ischemic stroke N Engl J Med 372, 2296-2306 
Kidwell CS, Chalela JA, Saver JL, Starkman S, Hill MD, Demchuk AM, Butman JA, Patronas N, Alger JR, Latour LL et al. (2004): Comparison of MRI and CT for detection of acute intracerebral hemorrhage JAMA $\underline{292}$, 1823-1830

Kidwell CS, Jahan R, Gornbein J, Alger JR, Nenov V, Ajani Z, Feng L, Meyer BC, Olson S, Schwamm LH et al. (2013): A trial of imaging selection and endovascular treatment for ischemic stroke N Engl J Med $\underline{368}$, 914-923

Kim JT, Park MS, Choi KH, Nam TS, Choi SM, Lee SH, Kim BC, Kim MK, Cho KH (2010): The CBV-ASPECT Score as a predictor of fatal stroke in a hyperacute state Eur Neurol $\underline{63}, 357-363$

Kucinski T (2005): Unenhanced CT and acute stroke physiology Neuroimaging Clin N Am $\underline{15}, 397-407$, xi-xii

Lansberg MG, Straka M, Kemp S, Mlynash M, Wechsler LR, Jovin TG, Wilder MJ, Lutsep HL, Czartoski TJ, Bernstein RA et al. (2012): MRI profile and response to endovascular reperfusion after stroke (DEFUSE 2): a prospective cohort study Lancet Neurol 11, 860-867

Larrue V, von Kummer RR, Muller A, Bluhmki E (2001): Risk factors for severe hemorrhagic transformation in ischemic stroke patients treated with recombinant tissue plasminogen activator: a secondary analysis of the European-Australasian Acute Stroke Study (ECASS II) Stroke $\underline{32}$, 438-441

Lee JH, Kim YJ, Choi JW, Roh HG, Chun YI, Cho HJ, Kim HY (2013): Multimodal CT: favorable outcome factors in acute middle cerebral artery stroke with large artery occlusion Eur Neurol 69, 366-374

Lum C, Ahmed ME, Patro S, Thornhill R, Hogan M, Iancu D, Lesiuk H, Dos Santos M, Dowlatshahi D (2014): Computed tomographic angiography and cerebral blood volume can predict final infarct volume and outcome after recanalization Stroke $\underline{45}, 2683-2688$

Mak HK, Yau KK, Khong PL, Ching AS, Cheng PW, Au-Yeung PK, Pang PK, Wong KC, Chan BP (2003): Hypodensity of $>1 / 3$ middle cerebral artery territory versus Alberta Stroke Programme Early CT Score (ASPECTS): comparison of two methods of quantitative evaluation of early CT changes in hyperacute ischemic stroke in the community setting Stroke $\underline{34}$, 1194-1196

Nogueira RG, Jadhav AP, Haussen DC, Bonafe A, Budzik RF, Bhuva P, Yavagal DR, Ribo M, Cognard C, Hanel RA et al. (2018): Thrombectomy 6 to 24 Hours after Stroke with a Mismatch between Deficit and Infarct N Engl J Med 378, 11-21 
Pexman JH, Barber PA, Hill MD, Sevick RJ, Demchuk AM, Hudon ME, Hu WY, Buchan AM (2001): Use of the Alberta Stroke Program Early CT Score (ASPECTS) for assessing CT scans in patients with acute stroke AJNR Am J Neuroradiol 22, 1534-1542

Psychogios MN, Schramm P, Frolich AM, Kallenberg K, Wasser K, Reinhardt L, Kreusch AS, Jung K, Knauth M (2013): Alberta Stroke Program Early CT Scale evaluation of multimodal computed tomography in predicting clinical outcomes of stroke patients treated with aspiration thrombectomy Stroke $\underline{44}, 2188-2193$

Saver JL, Goyal M, Bonafe A, Diener HC, Levy EI, Pereira VM, Albers GW, Cognard C, Cohen DJ, Hacke W et al. (2015): Stent-retriever thrombectomy after intravenous t-PA vs. t-PA alone in stroke N Engl J Med 372, 2285-2295

Schramm P, Schellinger PD, Klotz E, Kallenberg K, Fiebach JB, Kulkens S, Heiland S, Knauth M, Sartor K (2004): Comparison of perfusion computed tomography and computed tomography angiography source images with perfusion-weighted imaging and diffusion-weighted imaging in patients with acute stroke of less than 6 hours' duration Stroke $\underline{35}, 1652-1658$

Schregel K, Behme D, Tsogkas I, Knauth M, Maier I, Karch A, Mikolajczyk R, Hinz J, Liman J, Psychogios MN (2016): Effects of Workflow Optimization in Endovascularly Treated Stroke Patients - A Pre-Post Effectiveness Study PLoS One 11, e0169192

Sharma M, Fox AJ, Symons S, Jairath A, Aviv RI (2011): CT angiographic source images: flow- or volume-weighted? AJNR Am J Neuroradiol 32, 359-364

The National Institute of Neurological Disorders and Stroke rt-PA Stroke Study Group (1995): Tissue plasminogen activator for acute ischemic stroke. N Engl J Med 333, 15811587

Trenkler J (2008): Acute ischemic stroke. Diagnostic imaging and interventional options Radiologe $\underline{48}, 457-473$

von Kummer R, Meyding-Lamade U, Forsting M, Rosin L, Rieke K, Hacke W, Sartor K (1994): Sensitivity and prognostic value of early CT in occlusion of the middle cerebral artery trunk AJNR Am J Neuroradiol 15, 9-15; discussion 16-18

Wardlaw JM, Mielke O (2005): Early signs of brain infarction at CT: observer reliability and outcome after thrombolytic treatment--systematic review Radiology 235, 444-453 
Wiesmann M, Bohner G, Klingebiel R (2004): Zerebrale Perfusionsbildgebung mittels Mehrschichtspiral-CT Clin Neuroradiol 14, 92-107 


\section{Kopie der Publikation}

Fur Radol

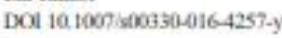

NEURO

\section{Added value of CT perfusion compared to CT angiography in predicting clinical outcomes of stroke patients treated with mechanical thrombectomy}

loannis Tsogkas ${ }^{1}$, Michael Knauth ${ }^{1}$, Katharina Schregel ${ }^{1}$, Daniel Behme ${ }^{1}$,
Katrin Wasser $^{2}$, Ilke Maier ${ }^{2}$, Jan Liman ${ }^{2}$. Marios Nikos Psychogios ${ }^{1}$

Received. 7 July 2015/Revised. 18 January 2016/Acocpeat: 28 Jamuary 2016 E) Euspean Society of Radiology 2016

\begin{abstract}
Objectives CIP images analyzed with the Alberta strulke program early CT scale (ASPECTS) have been shown to be op. timal predictors of elinical outcome. In this study we com pared two biomarkers, the cerebral blood volume (CBV) ASPECTS and the CTA-ASPECTS as predictors of clinical outcome after thrombectorny.

Methods Stroke patients with thrombosis of the MI segmeat of the middle cercbral artery were included in our study. All patients underwent initial multimodal CT with CTP and CTA on a modern CT scanner. Treatment consisted of full dose intravenous tissue plasminogen activator, when applicable, and mechanical thrombectony. Three neuroradiologists sepo. rately scored CTP and CTA images with the ASPECTS score. Results Sixty-five patients were included. Median baseline CBV-ASPECTS and CTA-ASPECTS for patients with fivourable clinical outcome at follow-up were 8 [interquartile range (IQR) 8.9 and 7.9 respectively]. Patients with poor clin ical outcome showed a median baseline CBV.ASPECTS of 6 (IQR 5-8, P $<0,0001$ ) and a median baseline CTA-ASPECTS of 7 (IOR 7-8, $P=0.18$ ). Using CBV-ASPECTS and CTA. ASPECTS raters predicted futile reperfusions in $96 \%$ and $56 \%$ of the cases, respectively.

Conclusions CBV-ASPECTS is a significant predictor of clinical veteome in patients with acuse ischemic stroke treated with mechanical thrombectomy.
\end{abstract}

20. Marios Niluos Psychogivo

inpsychogionin mad uni-goestingende

Depunnert of Neuronaliology, Unwersiny Meticine Goeningen, Rohert Koch 40, 37075 Goctingen, Gemuny

2 Deputrent of Neurolory. Unwasiny Medicine Goettingan, Goetringen, Gemamy
Key Points

- CBV ASPECTS is a significant predictor of climical outcome.

- Single phase CTA-ASPECTS has low protictive valine.

- Using CBV:ASPECTS, raters identified futile reperfiesions in $96 \%$ of the cuases.

Keywords Neurvimsging + X-ray computed - Stroke

Thrombectomy - Patient selection

\section{Abbreviations}

ASPECTS Alberta stroke program early CT score

CBF

CBV

GSS

ICC

IQR

IV rPA Intravenous recombinant tixsue plasminogen activator

MCA Middle cerebral artery

mTICI Modified thrombolysis in cerehral infarction

MTT Mean transit time

mRS Modified Rankin scale

NiHSS National lnstitutes of Health stroke scale

NCCT poncontrast computed tomography

NPV Negative predictive value

PPV Positive predictive value

TTD Time to drain

Introduction

Patient selection is essential for the improvement of elinical outcome in treating stroke patients with mechanical thrombectomy [1, 2]. Until publication of the latest 
randomized trials, studies had shown the feasibility of mechanical thrombectomy in restoring perfusion distal to the initial occlusion site, but had failed to report a substantial increase in favourable clinical outcome $[3,4]$. Even in the latest randomized trial, which proved the superiority of com hined mechanical throenboctomy and intravenous thromboly. six versus intravenous thrombolysis alone, functional independence was observed in only $32.6 \%$ of treated patients after 90 days [5]. In a recent study, it was suggested that cerebral blood volume (CBV) maps, evaluated with the Alberta stroke program carly CT score (ASPECTS), are strong predictoes of outcome [6]. Hence, selection of patients based on CTP can reduce futile reperfusions. In this study we compared the CBV-ASPECTS with the CTA-ASPECTS as final outcome predictors in a highly select group of stroke patients treated with mechanical thrombectomy. We additionally introduced a novel scoring system combining netrological and CTP. findings as well as age to further improve the selection process.

\section{Materials and methods}

Patients were retrospectively identified from our university hoopital's institutional tevicw bound-appeoved stroke database according to the following inclusion criterix

1. Acquisition of a complete multi-modal CT examination between January of 2011 and December of 2014 including noncontrast computed tomography (NCCT) of the head, near whole-hrain CTP, and single phase CTA of the bead and nock.

2. Presence of MI ocelusion on initial angiogram, as defined by modified thrombolysis in cerebral infarction score (mTICI) of 0 or I, a scale used to grade teperfusion of distal tissue bed [7]- Carotid bifurcation occlusions were exchuded from our study.

3. Successful reperfiusion of the middle cerebral artery as defined by mTICI $2 \mathrm{~b}$ or 3 on the final angiogram after mechanical thrumbectomy.

Exclusion criteria were incomplete CTP datasets in our department's picture archiving system, datasets with severe motion artefacts, patient age $<21$ years and reperfusion of the middle cerchral artery on initial angiograms.

Stroke imaging was acquired with a 128 -slice multidetector CT scanner (Siemens Definition ASt; Siemens Healtheare Sector, Forchheim, Germany) and included a NCCT, followed by near whole-brain CTP and single phase CTA of extra- and intracranial arteries. CTP consisted of 30 consecutive spiral scans of the brain (96 mm z-axis coverage, $2 \mathrm{~s}$ delay after start of contrast agent injection, 45 s total acquisition time, $80 \mathrm{kV}$,
$200 \mathrm{mAs}$ and effective dose of $-5 \mathrm{mSv}$ ). Thirty-six $\mathrm{mL}$ of contrast agent were injected with a rate of $6 \mathrm{~mL} / \mathrm{s}$ through a cubital vein, followed by $30 \mathrm{~mL}$ of sline chas. er. CTP data were reconstructed with a slice thickness of $5 \mathrm{~mm}$ every $3 \mathrm{~mm}$ (H20f Kernel, 512 matrix) and were further processed by a neuroradiologist using a commercial analysis package (Volume Perfusion CT Neuro; Siemens) with a delay-invariant deconvolution method, automatic motion correction and a dedicated noise reduction technique for dynamic data. For CTA $(120 \mathrm{kV}, 120$ reference $\mathrm{m} / \mathrm{As}, 0.3 \mathrm{~s}$ rotation time, 0.6 pitch, $2 \times 64 \times 0.6 \mathrm{~mm}$ collimation, $-3 \mathrm{mSv}$ ) $45 \mathrm{ml}$ of contrast agent were injected, followed by $30 \mathrm{~mL}$ of saline chaser. CTA was acquired with a single phase protocol using bolus triggering in the aortic arch (100 HU threshold; $5 \mathrm{~s}$ delay for bolus watch, 3 s delay after reaching thresholdh. CTA data were them reconstructed to multiplanar images with a section width of $5 \mathrm{~mm}$ and increment of $3 \mathrm{~mm}$.

According to protocol, patients presenting within 4.5 hours of symptom onset with no significant infurction or haemorrhage detected on NCCT were eligible for intravenous recombinant tissue plasminogen activator (IV rPA). IV rPA ( $0.9 \mathrm{mg} / \mathrm{kg}$ over $60 \mathrm{~min})$ was then administered directly after noncontrast CT. Following stroke imaging, patients were transferred to the angiography suite (Artis Zee, Siemens Healthcare, Forchheim, Germany) where they underwent mechanical thrombectomy either with a Penumbra MAXTM reperfusion eatheter (Penumbra, Alameda, California) of with a combination of aspiration with reperfusion catheters and retrievable stents (Aperio, Acandis, Pforzheim, Germany; Trevo, Stryker, Mountain View, California; 3D Sepurator, Penumbra). In cases of rapid transport to angiography and fast mechanical thrombectomy the remaining rtPA was administred intraarterially either before or after recan. alization at the discretion of the interventional neuroradiologist. Appropriate anaesthesia (intravenous conscious sedation or general anaesthesia) was obtained as per stan. dard practice

All data used in this study (demographic dath, vascular risk factors, mTICI scores, various times, and neurological scores) were extracted from the aforementioned datahase. Neurological scores (National Institutes of Health Stroke Scale (NIHSS), modified Rankin scale (mRS), etc) were assessed by a certified stroke neurologist at hospital admission, bospital discharge, and discharge from the rehabilitation unit. Interventional and stroke imaging scores were assigned in consensus by three neurveadiologists (two with more than 5 years of experience). The neuroradiologists separately rated NCCT, CTP, and CTA scans with the ASPECTS, a 10-point scoring system of the middle cerebral artery (MCA) ternitory. For every MCA region with acute ischemic signs, 1 point is subtracted from 10 , 
resulting in an ASPECTS of 10 for a scan without ischemic lesions and an ASPECTS of 0 for complete MCA. infarctions (www.aspectsinstroke.com, Fig. 1)

We also calculated a new scone, the Goettinger stroke scale (GSS) according to the following formula: CBV.ASPECTS (NIHSS/4 + age/20)

The introduction of this novel score was based on the ob. servation that each of the parameters admission NIHSS, age, and CBV-ASPECTS already predicted outeome fairly well on its own. A combination of all three should have the potential to further improve predictability.

Seans of every patient were divided into two groups, which were rated with a 90-day gap in between: At first NCCT scans were rated simultaneously with CTA images; the second group consisted of coloured CTP parameter maps including cerebral blood flow (CBF), CBV, mean transit time (MTT), and time to druin (TTD) maps. The latter is a very sensitive parameter depicting the total extent of perfusion lesions [8]. Additionally, raters were retrospectively asked to prediet futile reperfiusions bassed either on NCCT, CTA or on CTP images. NCCT/CTA-ASPECTS, or CBV-ASPECTS lower than 7 was the primary criterion for futile reperfusions in the groups. Cases of disagreement were settled by consensus. Raters were blinded to all clinical outcomes.

Study parameters were compared between patients with follow-up $m R S>2$ (poor outcome) and with follow-up mRS $\leq 2$ (favorable outcome). In cases with mixsing follow-up mRS values, discharge mRS was ussed instead [9]. Continuous po. rameters were compared either with the Welch $\mathrm{t}$ test, in cases of nommal distribution, or with the Mann-Whitney U test, in case of nom normsal or ondinal distribution. Categorical vari ables were compared hetwean the two groups by the Fischer exact test. Additionally, we calculated the probability of good outcome at follow-up by stepwise logistic regression using all variables that were significantly different between groups in the univariate analysis. Selected variables were further examined by receiver operating characteristic analysis. Interobserver agreement was calculated with the interclass correlation coefficient (ICC). Analyses were performed with the MedCalc statistical package (MedCalc, Mariakerke, Belgium). The significance level for all tests was set to $a=0.05$.

\section{Results}

Of the 72 patients identified, seven were excluded because of incomplete imaging data sets. Sixty-five paticnts were incladed for analysis (26 women; median age 72 years; interquartile range (IQR) 68-76). Median NIHSS at admission was 17 (IQR, 12-21) and median mRS at admission was 5 (IQR, 45). All patients had suxcessful reperfusion on firsal angiograms (mTIC12b or 3). In the majority of cases (56\%) mechunical thrombectorny was combined with IV and IA rePA therapy; IV rtPA and mechanical thrombectomy was coembined in $26 \%$. with sole mechanical thrombectony accounting for $18 \%$ of cases. At discharge, NIHISS was reduced to a modian of 7 (TQR, 4-12) and mRS to a median of 4 (IQR, 2-5). The median follow-up mRS was 3 (IQR, 2-4). Overall, $40 \%$ of our patients had a favourahle outcome at follow-up.

of the analyzed medical comorhiditics, only diabetes mellitus $(P-0.013)$ and obesity $(P=0.04)$ were significantly different between patients with favourable and poor outcome (Table 1). Reganding imaging variables, the CBVaSPECTS
Fì. 1 NCCT images depicting the ASPECTS score Oee point is sobrasted froes ter for any signs of earty iachemia in the definod regioms. ASPECTS is scored in Two regioes of the MCA territory. the busal endia level and the sqpragraglininic kvel
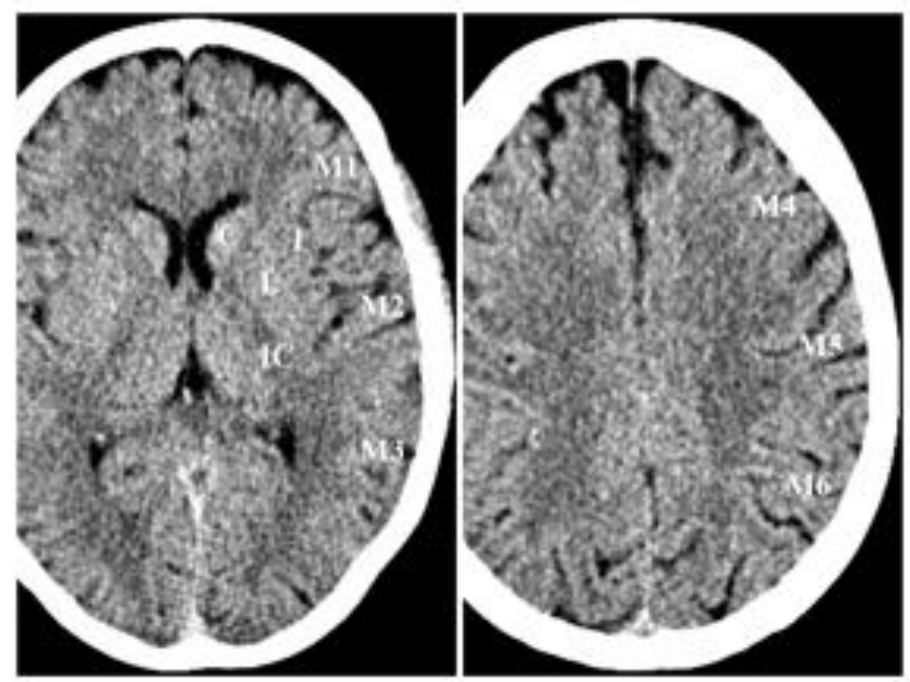
Table 1 Baseline characieristicx, ueatunent times, and climical outoone betwect patients with frvonable and poor ouboome at follow-up

\begin{tabular}{|c|c|c|c|c|}
\hline & Total $(n-65)$ & $\begin{array}{l}\text { Follow-up mRS } S \\
(n=26)\end{array}$ & $\begin{array}{l}\text { Follow-ip mRS }>2 \\
(w=39)\end{array}$ & $p$ \\
\hline Ags, medim (IOR) y & $72(68-76)$ & $71.5(59-75)$ & $73(69-78)$ & 0.1t \\
\hline Admiscisa NLHSS, median (IXYR) & $17(12-21)$ & $14(10-17)$ & $18(15.24)$ & $0.0003^{*}$ \\
\hline Admission mRS, modie (IQR) & $5(4-5)$ & $4(4-5)$ & $5(5-5)$ & $40.0001^{\circ}$ \\
\hline \multicolumn{5}{|l|}{ Medical comotbatities } \\
\hline Hyperlipidentaia & $42(65 \% 6)$ & $17(65 \%)$ & $25(64 \%)$ & 1 \\
\hline Hypenension & $57(88 * 4)$ & $21(81 \%)$ & $36(92 \%)$ & 0.25 \\
\hline Diabetes mellitus & $19(30 \%)$ & $3(12 \%)$ & $16(41 \%)$ & $0.013 *$ \\
\hline Sensking & $18(30 * 6)$ & $7(30 \%)$ & $11(30 \%)$ & 1 \\
\hline PMD & $2(3 *)$ & $1(4 \%)$ & $1(3 \%)$ & 1 \\
\hline Obesiny & $33(55 \%)$ & $9(38 \div 9)$ & $24(67 * 4)$ & $0.04^{*}$ \\
\hline \multicolumn{5}{|l|}{ Imaging scores, modin (IQR) } \\
\hline CT $A S P E C T S$ & $9(8.9)$ & $9(9-9)$ & $8(7-9)$ & $0.03^{*}$ \\
\hline CTA ASAECTS & $7(7-8)$ & $8(7-9)$ & $7(7-8)$ & 0.18 \\
\hline CBV ASPECTS & $8(6-8)$ & $8(8-9)$ & $6(5-8)$ & $0.0001^{*}$ \\
\hline CPE ASPECTS & $4(3-5)$ & $4(4-5)$ & $4(3-5)$ & 0.27 \\
\hline TTD ASPECTS & $2(1-3)$ & $2.5(1.3)$ & $2(1-3)$ & 0.53 \\
\hline MTT ASPECTS & $3(1.75-3)$ & $3(2.3)$ & $3(1-3)$ & 0.62 \\
\hline$\triangle($ (CBVCBF) ASPECTS & $3(1-4)$ & $4(3-5)$ & $3(1-3)$ & $0.0014^{*}$ \\
\hline$\triangle(C B V-T T D)$ ASPECTS & $5(4-6)$ & $6(5.7)$ & $4(3-6)$ & $0.0012^{*}$ \\
\hline$\triangle($ CBVMTT $)$ ASPECTS & $4(3-6)$ & $5.5(4-6)$ & $4(3-5)$ & $0.000 s^{*}$ \\
\hline GSS & $-0.05(-2.6-1.65)$ & $1.6(0.25-2.4)$ & $-1.5\{-3.6--0.01\}$ & $40.0001^{*}$ \\
\hline Orset $00 \mathrm{minC} 2 \mathrm{~b}$, modien (IQR) & $254(222-317)$ & $237(205-306)$ & $261(235.319)$ & 0.16 \\
\hline Gimin so mincL $2 \mathrm{~b}$, medim ( $\mathrm{QQR}$ ) & $51(35-70)$ & $49(35-77)$ & $51(33-49)$ & 0.91 \\
\hline Discharge NHSS, modin (OQR) & $7(4-15)$ & $3(1-5)$ & $13(9-20)$ & $40.0001^{*}$ \\
\hline Dischunge mRS, mediun (IOR) & $4(2-5)$ & $1.5(1.2)$ & $5(4-5)$ & $-40001^{\circ}$ \\
\hline Follow-cp mRS, median (IOR) & $3(2-4)$ & $1(t-2)$ & $4(4-5)$ & 4) $0001^{\circ}$ \\
\hline
\end{tabular}

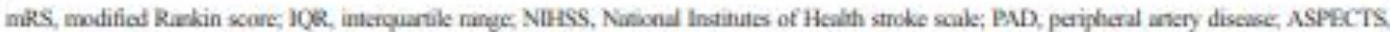
Alberta suoke program early CT scale, CTA, CT angography; CBV, cerebcal bibod voluene, CBF, cerchral blood flow; TTD, tine to drain; MTT, mexn transit time; CSS, Gocnirger stroke scale. TIC1, thowholysis in cerchral infunction

$+P$ value sigrificant

and the various mismatch scores achieved high significance levels with $P=0.0014$ and $P<0.01$, respectively (Table 1 ). NCCT-ASPECTS and GSS values were also significantly dif ferent between the two groups with $P=0.03$ and $P<0.0001$, respectively. Patients with favourable outcome had a baseline CTA-ASPECTS of 8 (IQR, 7-9), while patients with poor follow-up outcome had a baseline CTA-ASPECTS of 7 (IQR, $7-8 ; P-0.18$ ). There was no significant difference in time frum symptom onset or groin puncture to successful reperfusion between the two groups $(P=0.16$ and $P=0.91)$.

When asked to predict futile reperfiusions basod on NCCT and CTA imiges, raters were able to recognize $56 \%$ of the patients with poor outcoene. The percentage was significantly higher when raters based their decisions on CTP images, as they were able to recognize poor outevenes in $96 \%$ of the cases (Table 2). CBV-ASPECTS based decisions were also better for the prediction of favourable outcome, as raters recognized $61 \%$ of patients with favourable outcome as opposed to $39 \%$ of the cases with CTA-ASPECTS based decisions. Receiver operating characteristic analyses showed similar results. Sensitivity, specificity, positive predictive and negative predictive values (PPV, NPV) for NCCT-ASPECTS $<7$ were $8 \%, 100 \%, 100 \%$, and $42 \%$. For CTA-ASPECTS $<7$ sensitivity, specificity, PPV and NPV were $23 \%, 77 \%, 60 \%$, and $40 \%$. CBV.ASPECTS showed an optimal criterion value of $<7$ with a sensitivity of $49 \%$, specificity of $96 \%$, PPV of $95 \%$ and $\mathrm{NPV}$ of $56 \%$ (Fig. 2 ).

In the stepwise logistic regression analysis, only admission NIHSS $(P-0.04$; odds ratio $1.14 ; 95 \%$ confidence interval [C] 1.00 to 1.31) and CBV-ASPECTS $(P=0.006$; odds ratio $0.45 ; 95 \%$ CI0 0.26 to 0.79 ) were significant contributors to the prediction of outcome. Agreement between the three raters was very good with ICC values of 0.92 for CBV-ASPECTS (95\% Cl, 0.89-0.95), 0.9 for CBF ASPECTS ( $95 \%$ Cl, 0.85- 
Fur Radol

Tabie 2 Retmspoctive prediction of ostome bosed on beseline NCCT-ASPECTS, CTA.ASPECTS, or CBV-ASPECTS

\begin{tabular}{|c|c|c|c|c|}
\hline & Ruters' opinire & $\begin{array}{l}\text { Follow-ep mRS } \\
2 n=26\end{array}$ & $\begin{array}{l}\text { Follow-up miks } \\
>2=39\end{array}$ & $\begin{array}{l}\text { Finher's exact } \\
\text { test } P \text { value }\end{array}$ \\
\hline Dexision based on NCCT-ASPECTS & $\begin{array}{l}\text { Futile reperfinsion } \\
\text { Usefil reperiasion }\end{array}$ & $0 \%$ & $\begin{array}{l}100 \% \\
57 \%\end{array}$ & 0.07 \\
\hline Decision hased on CTA.ASPECTS & $\begin{array}{l}\text { Furile ieperfision } \\
\text { Usefil repertision }\end{array}$ & $\begin{array}{l}44 \% \\
39 \%\end{array}$ & $\begin{array}{l}36 \% \\
61 \%\end{array}$ & 0.77 \\
\hline Decision based an CBV-ASPECTS & $\begin{array}{l}\text { Funle erperfusion } \\
\text { Usefill repertision }\end{array}$ & $61 \%$ & $\begin{array}{l}96 \% \\
39 \%\end{array}$ & $40.000 t$ \\
\hline
\end{tabular}

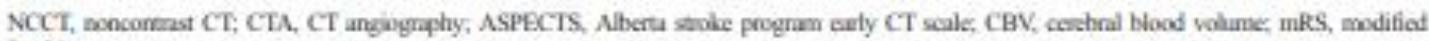
Rankin soore

0.93), 0.7 for NCCT-ASPECTS $(95 \% \mathrm{Cl}, 0.59-0.79), 0.88$ for CTA-ASPECTS ( $95 \% \mathrm{Cl}, 0.82-0.92), 0.71$ for MTT. ASPECTS $(95 \% \mathrm{Cl}, 0.6-0.8)$ and 0.85 for TTD-ASPECTS $(95 \% \mathrm{Cl}, 0.78-0.9)$.

\section{Discussion}

Our results support poeviously published studies supporting the uve of CBV-ASPECTS as a biomsarker in acute ischemic stroke treatment $[10,11]$. CBV.ASPECTS was significantly different in the univariate analysis and a significant predictor of outcome in the logistic regression model with $P=0.006$ and an odds ratio of 0.45 . When using CBV-ASPECTS, raters were able to identify futile reperfusions in $96 \%$ of the cases. The other significant predictor of outcome was the admission NIHSS. Our results disagree with previous publications show: ing that CTA-ASPECTS, acquired with a modern fast CT

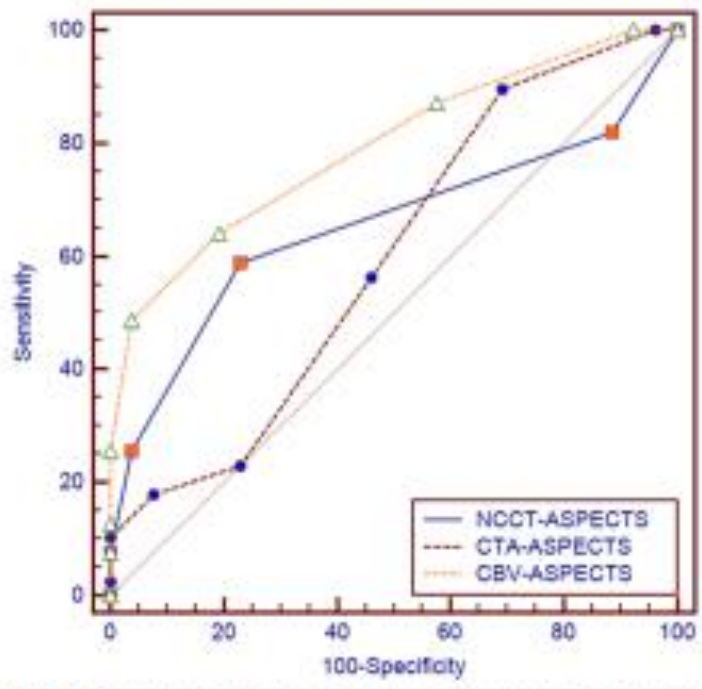

Fiz. 2 Comparison of roceiver operating chanacteristic analyxes of NCTT-CTA and CBV.ASPECTS scanner, is a significant predictor of outcomse (Fig. 3) [12]. It is worth mentioning that CTA images in the publication by Lum et al. were extracted out of a CTP dataset, using a predefinod point on the contrast-time curve. In our study we used a single phase acquisition protocol for CTA with a bolusadapted delay, which is a widely used protocol for the detertion of large vessel oeclusion. Studies with older, slower scanners have shown no significant differenoe in volume lesion between CIA and CBV images [13]. With the introduction of fast multisection scanners there have been reports that CTA images are $\mathrm{CBF}$ - rather than CBV-weighted, thus overestimating infarct core [14]. Our results support these findings, as CTA-ASPECTS was not significantly different between patients with favourable and poor outcomes and was not a significant predictor of outcome in the logistic regression model. When asked to retrospectively predict futile reperfusions based on CTA images, raters were successful in $56 \%$ of the cases.

Five randomined trials published in the last months have shown the superionity of endowaseular treatment compared to IV lysis alone in the treatment of stroke patients with large vessel occlusions $[5,15-18]$. The rate of favourable 90-day outcome after endovascular treatment varied from $33 \%$ (MRCLEAN) to $71 \%$ (EXTEND-1A). The trial with the lowest percentage of favourable outcomes had the widest inclession crituria and did not require demonstratiog of a small core on imaging, while the trial with the highest percentage used CTP to select patients. The great difference in favourable outcome percentages emphasizes the importance of proper imiging for patient selection and reduction of futile reperfiusions. Using CBV-ASPECTS as a selection criteriva has led to a significant increase in favourable vatcomes in our department the last two years, with $34 \%$ of favourable outcomes between 2007 and 2010 compared to $60 \%$ of good outcomes from 2012 to 2014 [3]. We increased the median CBV-ASCPECTS by 2 points during this time frame with a median of 6 in 2007-2010 and a median of 8 in 2012.2014 [6]. After publication of the aforementioned trials in 2015 we stopped using CBV-ASPECTS as a selection criterion for thrombectsmy treatment within the first 6 hours after symptom onset. However, we still use a 

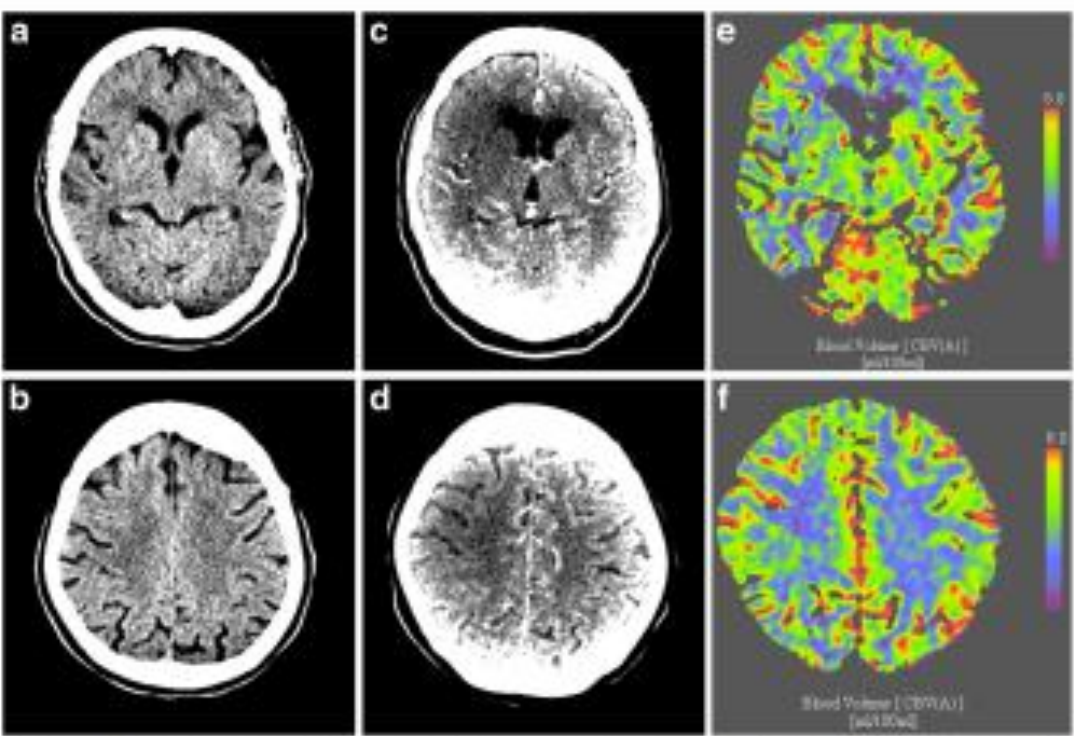

Fip. 3 lmages of a 75-yex-old woman presenting with an abmission NHHSS of 16 and admission miks of 3. NCCT scan fa. b) staws initial signs of eerebeal ischemia in the right insular conter and aas scored with an NCCT-ASPECTS of 9. CTA imanes (C, D) depict hypodense regions of the right caudate and lentiform nucles, insular ribeon and fromal operculum resuling in a CTA-ASPECTS of 6. CBV inages (E, F) stow

reduction of the ceretbal Wlood wolume oe the right insolur nbbon and were scored with a CBV-ASPECTS of 9 . After swift and successfit mechunical thromboctomy of the Mi ocdusion (groin to TiC Izb time of 45 minutes) patient showad significant improvement of ber clinical sympoens and was discharged with and mRS of 1

CBV-ASPECTS $>6$ to select patients for mechanical thrombectomy in the 6 to 12 hour window after stroke symptoen onset or in case of wake-up stroke.

The main argument against a tight selection strategy in treating acuse stroke patients with large artery occlusions is the reduction of the number of patients treated with the most effective therapy. Untreated patients may still have profited from treatment, but at a lower percentage than those with a positive imaging profile. Arvund $30 \%$ of patients with large vessel occlusions that would have peobably boen randonized in the NCCT-based trials were not included in EXTEND-LA based on imaging characteristies. This fact reduces the value of high percentage of favourable outeomes in a tight selection environment, as the absolute number of patients with favourable outcomes is probably the same as in the MR. CLEAN paradigm. The relevant difference, as shown by the shift analysis in MR-CLEAN, is the consistent shift towards better outcomes in favour of the intervention for all eategories of the mRS.

In our study, the only patient with CBV-ASPECTS $<7$ and favourable outcome after successful reperfusion was a young patient (27y) with a moderate admission NIHSS score (10). This finding led us to the description of the GSS, a score combining adnission NIISS and CBV.ASPECTS, as strong predictors of outcome, together with age. The GSS was sta. tistically different $(P<0.0001)$ between patients with favourable and poor clinical outcome with a median of 1.6

(IQR $0.25-2.4$ ) and -1.5 (IQR $-3.6--0.01$ ), respectively. Although initial results are promising, the predictive or com: plementary value of this soore has to be validated in further stadies. Other authors have proposed the conbination of multiple dagnostic parameters in a new score for the prediction of futile recanalizations [19]. However, as CTA collaterals and CT perfusion imaging potentially depict the same biological feature about ischemic tissue sustenance [20], we are not sure about the feasibility of the score proposed by Espinosa de Rueda et al. Physicians shocald also be aware of the potential risk of CT collateral scoring on single-phase CTA, as singlephase acquisitions are dependent on bolus timing and can underestimate collateral state. This risk can be eliminated with use of multiphase CTA [21].

The primary limitation of our study is the retrospective character of our analysis and the highly select group of patients. Only patients with successful reperfusica were inchud. ed in our study. Data were exiracted from a prospectively collected database and consecutive patients were included in our analysis, bat CTP images were uxed in treatment decisions between September 2012 and December 2014. Noncontrast CT was the primary imaging selection criterion before September 2012. This imaging selection praxis probably explains the fact that time (either onset to recanalization or groin to recanalization) was not a significant predictor of outcome in our study. Our results may only apply to CTP data acquirsd and reconstructed with the specific protocols and software 
stated in methods, as other authors have found significant differences between various vendors [22]. Furthermore, al. though studies have presented better predictive value of com. bined CTP and CTA we did not analyze a combined ASPECTS seore of these variables [23].

Our study suggests that CBV-ASPECTS is a significant predictor of clinical outeome in patients with acube ischemic stroke treated with mechanical thrombectomy. We discourage the use of single phase CTA-ASPECTS, scquired with a moden fast multisection CT scanner, as an imaging biomarker because of its low predictive value. Obviously, our results do not apply to CTA images extracted out of a CTP scan or to a multiphase CTA protocol.

Adinemiedgments The sciensific garanoe of this peblication is De Marios Nikos Psychogios. The arators of this manuscrige declare so relirionships with any comparies, whose penducts or services may be rclated to the subject mutier of the article. The aurbors state that ith work has nos receival amy funding, One of the antbors has significant statistical expertise. Inaticional Review Boand approval was obtained. Wriner infamed consern was waived by the Institutional Review Bosed. We would tike to infoen you thes port of the currert porient collective fabost one-thid of the popeulation) has been subject to peevions analyses: Frolict

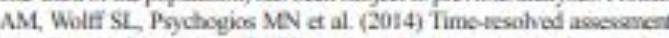
of collateral bow using $4 \mathrm{D}$ CT angograpby in large-vessel coclusioe sabke. Eur Radiol 24/2) 390-4. Methodology; retrospective, obscrvational, performed at one institution.

\section{References}

1. Cloft He (2011) Death and desaction in the irma-arterial hattle with acute ischenic stroke. ANR Am J Neuraratiol 32:1769-177

2. Lanherg MG. Sinka M, Kemp S es al (20t2) MRI protile and repponse to endovascular repertission after strobe (DEHLSE 2) a

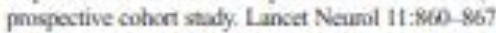

3. Prychogios MN. Kressech A. Wusser K. Motre A. Groschel K. Knuah M (2012) Recresaloation of large intracrarial vesecks using the pesumbra system: a single-center experiense. ANR Am Neumendiol 33:1408-1493

4. Nogueira RGi, Lussep HL, Grapa R et al (2012) Trevo varsus Mera retrievars for thombectomy revacularisation of large vessel occlu. sions in acute ischnemic stoble (TREVO 2) a nundomised trial. Lancet $380-1231-1240$

5. Berihemer OM, Fransen PS, Beamer D a al (2015) A randewsizod trial of intracterial treatment foe acute ischemic stroke. N Engl. Med 372:11-20

6. Psychogios MEN, Schramm P, Frobich AM et al (2013) Alberta Stroke Pargram Eacty CT Scale evaluation of multimodal coenput. od tomography in prodicting clinical outconxes of struke porients. treatad wath appination thrombectormy. Stroke 44:2188-2193
7. Zaiden 00 , Yoo N, Khatri P es al (201.5) Recommendutions ce angiograptic revascularisation grading standards for acuse ischemic stroke: a consensess stanemere. Sacke 44:2600-2663

x Abels B. Kloce E. Tomandl BF. Kloska SP, Lell MM (2010) Perfision CT in acune ischemic strobe a qualinative and quantiative compariscm of deocevolvion and muinum slope approsech ANR Am J Neuroradiol 31:1690-1098

9. Onbiagele B, Saner IL. (2010) Day-90 acuse ischanic stroke oulcomes can be derived from early functional activity level. Cerehernase Dis 29-50-56

10. Aviv Rl, Mandeboen 1, Chalvaboty S cr al (2007) Aberta stroke program earty CT sooring of CT perficion in early stroke visuali-

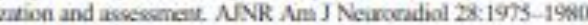

11. Kim J, Paik MS, Choe KH et al (2010) The CBV-ASPECT sone as a predictor of fand strobe in a hyperacuie sene. Eur Neard 63 ; $357-363$

12. Lum C. Ahmed ME, Proso S er al (2014) Conpuned tomographic angiograpty and cereheal blood volume can predict final infect volume and ourocnse after recamalization. Stroke 45:26K3-2685

13. Schinmen P, Schellinger PD, KJoes E et al $(2004)$ Compunison of pertision competed womograpty and compuned tomogrighy angiography source images with perfusion-weighted imaging and diffusion-weighnod insuging in poriens with acute stroke of less than 6 hours duration. Stmike 35:1652-1608

14. Shama M. Fox $\mathrm{N}$, Symoes $\mathrm{S}$, lairah $\mathrm{A}$, Aviv RI (2011) CT angiognaphic source images: flow- or volume-weighted? ANR Am J Neuroradiol 32:359-364

15. Goyd M, Demchol AM, Menon BK at al (2015) Randomizod issessment of rapid endovisocular treimuent of ischemic stable. N Engl J Med 372:1019-1030

16. Campbeli BC, Mindiel PJ, Kleinig TJ ef al (2015) Endovascular theripy for ischemic strobe with perfusion-imaing selection. $N$ Engl I Med 372:1009-1018

17. Saver JL, Goyal M, Bonafe $A$ et al (2015) Stemt-retriever thrombeckeny after irmavenous t-PA vs. $t$-PA abone in stroke. N Engl J Mad

18. Jovin TC, Chamomo A, Cobo Eet al (2015) Thecenbersony withis 8 bours afier sympoom onser in ischemic stroke. N Engl J Mod

19. Espinosa de Ruada M, Pamilla G, Manzano-Fermandex S et al (2015) Combined matrimodul conpated soemgraphy score comelates with futile recanalization afler throenhectomy is patiosts with acune stroke. Surobe 46c2517-2522

20. Vugal A. Menon BK, Fosier LO et al (2015) Askociation between $\mathrm{CT}$ angogram oollaterals and $\mathrm{CT}$ perfusion in the interventional management of stroke III trial. Sercike

21. Menon BK, dEstere CD, Qzxi EM et al (2015) Multiphuse CT angiograptry: a new lool for the imaging triage of patients with acute ischenic sroke. Radiology 275\$10-520

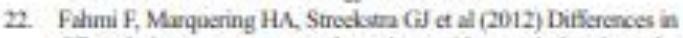
CT perfasion sumensy maps for paticnts with acule ischemic stroke generated by 2 sottware padages. ANRR Am I Neuroradol 33 . $2074-2000$

23. Lee JH, Kim YJ, Choi JW at al (2013) Mahimodal CT: favonable oatoone fastors in acute middle cendenal artery stroke with luge artery occlusion. Eur Neund 69-366-374 


\section{Danksagung}

Ich bedanke mich herzlich bei Herrn Prof. Dr. Marios-Nikos Psychogios dafür, dass er die Durchführung der hier vorliegenden Arbeit ermöglicht hat, sowie für die umfassende Betreuung und die Einführung in das wissenschaftliche Arbeiten.

Danken möchte ich auch den Mitarbeitern der Abteilungen für Neuroradiologie und Neurologie für die Mitarbeit bei dem Erstellen der Datenbank.

Schließlich möchte ich mich bei unserem ehemaligen Abteilungsdirektor, Herrn Prof. Dr. Knauth, bedanken. 